\title{
Fully compensated Kondo effect for a two-channel spin $S=1$ impurity
}

\author{
G. G. Blesio, ${ }^{1}$ L. O. Manuel, ${ }^{1}$ A. A. Aligia,${ }^{2}$ and P. Roura-Bas ${ }^{2}$ \\ ${ }^{1}$ Instituto de Física Rosario (CONICET) and Universidad Nacional de Rosario, \\ Bv. 27 de Febrero 210 bis, 2000 Rosario, Argentina \\ ${ }^{2}$ Centro Atómico Bariloche and Instituto Balseiro, \\ Comisión Nacional de Energía Atómica, CONICET, 8400 Bariloche, Argentina
}

\begin{abstract}
We study the low-temperature properties of the generalized Anderson impurity model in which two localized configurations, one with two doublets and the other with a triplet, are mixed by two degenerate conduction channels. By using the numerical renormalization group and the noncrossing approximation, we analyze the impurity entropy, its spectral density, and the equilibrium conductance for several values of the model parameters. Marked differences with respect to the conventional one-channel spin $s=1 / 2$ Anderson model, that can be traced as hallmarks of an impurity spin $S=1$, are found in the Kondo temperature, the width and position of the charge transfer peak, and the temperature dependence of the equilibrium conductance. Furthermore, we analyze the rich effects of a single-ion magnetic anisotropy $D$ on the Kondo behavior. In particular, as shown before, for large enough positive $D$ the system behaves as a "non-Landau" Fermi liquid that cannot be adiabatically connected to a non-interacting system turning off the interactions. For negative $D$ the Kondo effect is strongly suppressed. While the model is suitable for the description of a single $\mathrm{Ni}$ impurity embedded into an $\mathrm{O}$ doped $\mathrm{Au}$ chain, it is a generic one for $S=1$ and two channels and might be realized in other nanoscopic systems.
\end{abstract}

PACS numbers: 73.23.-b, 71.10.Hf, $75.20 . \mathrm{Hr}$

\section{Introduction}

The Kondo effect, early found in metals containing magnetic impurities ${ }^{1,2}$, is also frequently observed in low dimensional systems. For instance, transport measurements through semiconducting ${ }^{3-9}$ and molecular ${ }^{10-20}$ quantum dots (QDs), at low enough temperatures, exhibit the Kondo phenomena. Here, the QD acts as a single magnetic impurity while the contacts play the role of metallic hosts.

The seminal work by Nozières and Blandin ${ }^{21}$ pointed out the crucial role that the impurity and the conduction host orbital structures play in the Kondo physics. Therefore, real systems are expected to be modeled by Kondo Hamiltonians where an arbitrary spin $S$ is screened by $n$ conducting channels (bands with different symmetry) of spin $s=1 / 2$, and the nature of the ground state depends on the relation between $S$ and $n$. For $n=2 S$ the models have Fermi liquid ground states, while for $n>2 S$ they correspond to non-Fermi-liquids and for $n<2 S$ the systems are singular Fermi liquids ${ }^{22}$. The existence of nonFermi-liquid ground states requires $S U(n)$ symmetry in the conducting channels, which is difficult to achieve in real systems. For instance, in the case of the $S=1 / 2$ two-channel model, the effect of symmetry-breaking perturbations was discussed by Sela et al. ${ }^{23}$ It is also found that the presence of magnetic anisotropy can drastically modify the low-temperature properties ${ }^{24-27}$.

The multiorbital Kondo physics can be found in molecular QDs, which have rich inner electronic structures with magnetic orbitals coupled in a such a way that the resulting spin is sometimes larger than the usual $s=1 / 2$. For instance, the underscreened Kondo effect, corresponding to the case $n<2 S$, has been experimentally and theoretically investigated for molecules with spin $S=$ $1^{14,15,19,24,28-31}$, and larger $\operatorname{spin}^{31-33}$. In other systems, the spin ${ }^{13,34,35}$ and also the anisotropy $D^{35,36}$ can be manipulated. On the other hand, a Co impurity in an Odoped Au chain behaves as a QD with $S=3 / 2^{25,26,37}$. In this system, two conducting gold channels $\left(5 d_{x z}, 5 d_{y z}\right)$, degenerate by symmetry, screen only two electrons of the Co atom $\left(3 d_{x z}, 3 d_{y z}\right)$, while the electron on the $3 d_{x y}$ orbital is unaffected by the $\mathrm{Au}$ bands. In the presence of a single-ion magnetic anisotropy $D S_{z}^{2}$, with $D>0$, the effective impurity spin becomes $S=1 / 2$ and the corresponding scenario was found to be the overscreened Kondo effect, $n>2 S(n=2, S=1 / 2)^{25,26,37}$.

A difference between the bulk systems and the low dimensional QD ones is that while in the former the fully screened scenario $n=2 S$ is frequent ${ }^{38}$, it seems rare in the latter. However, as stated above, there are several studies on nanoscopic systems with $S>1 / 2$ and also with degenerate orbitals ${ }^{25,37,39-41}$ so that this scenario is expected to appear in the future. In particular, recently a realization of the fully compensated high spin Kondo phenomena in a low-dimensional system has been proposed $^{42}$. It was shown that a $\mathrm{Ni}$ impurity, within an $\mathrm{O}$ doped Au chain, has two holes in the degenerate $3 d_{x z, y z}$ orbitals, coupled to $S=1$ because of a large Hund's interaction $^{31,42}$. The coupling between the $5 d_{x z, y z} \mathrm{Au}$ bands (which doped with $\mathrm{O}$ cross the Fermi level) and the $3 d_{x z, y z}$ of Ni states leads to a two-channel $S=1$ Kondo effect. A scheme of the system is presented in Fig. 1. Based on first-principles calculations, the electronic structure was studied and effective Anderson- and Kondo-like Hamiltonians were derived in Ref. 42. Using model parameters, an experimentally accessible Kondo temperature $T_{K} \sim 70 \mathrm{~K}$ was estimated (see Supplemen- 
tal Material of Ref. ${ }^{43}$ ). More recently we have shown that the model has a topological phase transition as a function of the impurity single-ion magnetic anisotropy $D^{43}$. However, so far, the general properties of the model have not been studied.

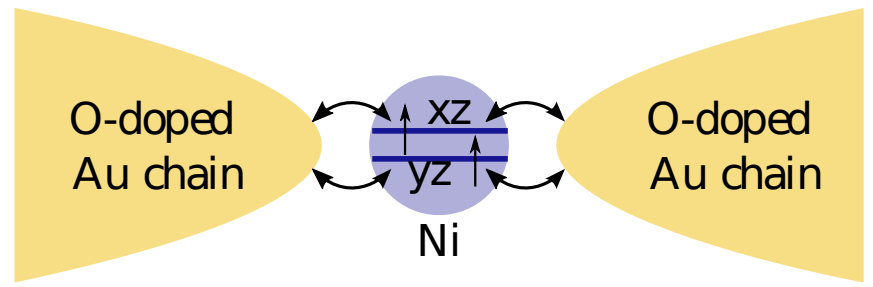

FIG. 1: Scheme of the system proposed in Ref. 42. The Ni ion has two holes, with total spin $S=1$, that can jump to conduction bands of the same symmetry either to the left or to the right.

While the Kondo screening of a spin $S=1 / 2$ has been the focus of an intense research since the $70 \mathrm{~s}$, both experimentally and theoretically, this is not the case of the full screening of the $S=1$ case. For instance, the longknown "Kondo resonance narrowing problem", that is, the exponentially decrease of the Kondo scale with the impurity spin $S^{44}$, has not received much attention until the recent work by Nevidomskyy and Coleman ${ }^{38}$.

In this work, in order to deepen the understanding of the high-spin fully compensated Kondo physics and to serve as a guide to experimental characterizations, we present calculations of the model presented in Ref. 42 for valence fluctuations between a configuration with one particle (two doublets) and another one with two particles (a triplet). This model corresponds to an impurity spin $S=1$ screened by two degenerate conduction channels. The particles can be electrons or holes. To solve the model, we use two complementary methods: the numerical renormalization-group (NRG) and the non-crossing approximation (NCA).

For $D=0$, we present results for the impurity entropy as a function of temperature $S(T)$, the impurity occupancy as a function of the impurity level $\epsilon_{d}$, the impurity spectral density $\rho(\omega)$ for different $\epsilon_{d}$ including a study of the position and width of the charge-transfer peak near $\epsilon_{d}$, the Kondo temperature as a function of $\epsilon_{d}$, and the conductance $S(T)$ as a function of temperature for several values of $\epsilon_{d}$. We also show how these results are modified by anisotropy $D$. In Ref. 43 we have shown some results for $S(T), \rho(\omega)$ and $G(T)$ for fixed $\epsilon_{d}$ and several $D \geq 0$, to show that a sharp jump in these properties take place at a given critical anisotropy $D_{c}$ due to a topological quantum phase transition. Here, we add new calculations in the Kondo limit particularly for $D$ near $D_{c}$ which illustrate the sharpness of the transition. We also study the case $D<0$. While for a $\mathrm{Ni}$ impurity in a $\mathrm{Au}$ chain a positive $D$ or the order of a few meV has been calculated $^{42}$, one might expect that experimental realizations of the fully screened Kondo model with $S>1 / 2$ and $D<0$ appear in the future. In particular negative tunable $D$ has been calculated in some systems containing phtalocyanine molecules ${ }^{35,36}$. The Haldane system $\mathrm{Y}_{2} \mathrm{BaNiO}_{5}$ has negative $D^{45}$ and one might expect the same for a $\mathrm{Ni}$ impurity in a similar environment.

For $D=0$, we have found, as expected ${ }^{21}$, several features that correspond to a Fermi liquid behavior at low enough temperatures, for example, the impurity entropy $S_{i m p}(T) \rightarrow 0$ as $T \rightarrow 0$. On the other hand, the impurity spectral density $\rho_{\alpha \sigma}(\omega)$ exhibits a single chargetransfer tunneling resonance (the impurity configuration with three particles is absent in the Hamiltonian), and also the Kondo one within the Kondo regime. The features of the resonances are discussed in comparison with the corresponding one-channel $S=1 / 2$ case. In particular, we have found that the temperature dependence of the conductance can be used as a hallmark to characterize a fully screened $S=1$ impurity. On the other hand, the presence of a single-ion magnetic anisotropy $D$ profoundly affects the Kondo physics. As we have shown recently for particular parameters ${ }^{43}$, for positive $D$ there is a topological transition at a critical value $D_{c}$ to another Fermi liquid phase which cannot be adiabatically connected to a non-interacting system. Instead for $D<0$, The Kondo physics is preserved but the Kondo energy scale is strongly reduced because the remaining two degenerate states of the localized triplet, with projections \pm 1 are mixed by an effective spin flip of fourth order in the hybridization between localized and conduction states.

The paper is organized as follows. In section II we introduce the model Hamiltonian as well as the NRG and NCA approaches. In section III the numerical solution of the model is presented, for several values of the model parameters including the particular case of the $\mathrm{Ni}-\mathrm{Au}-\mathrm{O}$ system considered in Ref. 42. Finally, in section IV the conclusions are drawn.

\section{Model Hamiltonian and Methods}

The Hamiltonian that describes the system of a magnetic $\mathrm{Ni}$ atom in a substitutional position within an $\mathrm{Au}$ chain doped with a small amount of oxygen can be written by using hole operators, $h_{\sigma}$, related with the electron operators, $d_{\sigma}^{\dagger}$, by $h_{\uparrow}=d_{\downarrow}^{\dagger}$ and $h_{\downarrow}=-d_{\uparrow}^{\dagger}$. This transformation preserves the form of the Hamiltonian and the spin operators, which have the same form in both representations. Using these operators for all atoms in the system, and retaining only the ground state of the $d^{8}$ and 
$d^{9}$ configurations, the model can be written in the form ${ }^{42}$

$$
\begin{aligned}
H & =\sum_{M_{2}}\left(E_{2}+D M_{2}^{2}\right)\left|1 M_{2}\right\rangle\left\langle 1 M_{2}\left|+\sum_{\alpha M_{1}} E_{1}\right| \alpha M_{1}\right\rangle\left\langle\alpha M_{1}\right| \\
& +\sum_{\nu k \alpha \sigma} \epsilon_{\nu k} c_{\nu k \alpha \sigma}^{\dagger} c_{\nu k \alpha \sigma}+ \\
& +\sum_{M_{1} M_{2} \nu k \alpha \sigma} V_{\nu \alpha}\left\langle 1 M_{2} \mid \frac{1}{2} \frac{1}{2} M_{1} \sigma\right\rangle\left(\left|M_{2}\right\rangle\left\langle\alpha M_{1}\right| c_{\nu k \alpha \sigma}+\text { H.c. }\right),
\end{aligned}
$$

where $E_{i}$ and $M_{i}$ indicate the energies and the spin projections along the chain (chosen as the quantization axis) of states with $i=1,2$ holes in the $3 d$ shell of the Ni impurity.

The ground state configuration of the $\mathrm{Ni}$ atom, described by the first term in Eq. (1) is found to have two holes in the degenerate $3 d_{x z, y z}$ orbitals coupled to spin $S=1$ by means of a strong Hund's coupling. The state with maximum spin projection can be represented by $|11\rangle=h_{x z \uparrow}^{\dagger} h_{y z \uparrow}^{\dagger}|0\rangle$, where $|0\rangle$ stands for the full $3 d^{10}$ configuration and the operator $h_{\alpha \sigma}^{\dagger}$ creates a hole with symmetry $\alpha=x z, y z$ and spin projection $\sigma$. The other relevant states of the $\mathrm{Ni} d^{8}$ configurations can be obtained by using the spin lowering operator on this state. $D$ represents the single-ion magnetic anisotropy.

The second term contains the relevant states of the $d^{9}$ configuration with $\left|x z M_{1}\right\rangle=h_{y z M_{1}}^{\dagger}|0\rangle$ and $\left|y z M_{1}\right\rangle=$ $h_{x z M_{1}}^{\dagger}|0\rangle$.

The third term represents the four conduction bands for the two channels $\alpha$ and conduction leads $\nu$. The operator $c_{\nu k \alpha \sigma}^{\dagger}$ creates a hole in the a Au-O band with symmetry $\alpha$, where $\nu=L, R$ denotes the left or the right side of the Ni atom, respectively.

The last term characterizes the tunneling between the $\mathrm{Ni}$ and $\mathrm{Au}$ states for each channel. The factor $V_{\nu \alpha}$ defines the hybridization for each channel and lead, and the Clebsch-Gordan coefficients $\left\langle 1 M_{2} \mid \frac{1}{2} \frac{1}{2} M_{1} \sigma\right\rangle$ determine the ratio among different angular momentum projections. For the calculations presented here, we take $V_{\nu \alpha}=V$ independent of lead and channel

Note that the presence of $\mathrm{O}$ atoms within the conducting chain is a necessary ingredient for the applicability of the model to the system, because electronegative $\mathrm{O}$ atoms deplete the $\mathrm{Au}$ bands and are responsible for the presence of $5 d_{x z, y z} \mathrm{Au}$ bands at the Fermi level ${ }^{25,26,37}$. With this condition, the tunneling mechanism responsible for charge transfer and spin-flip between $\mathrm{Ni}$ and $\mathrm{Au}$ neighbors is warranted.

\section{A. Numerical Renormalization Group}

In order to obtain quantitatively reliable results at low energies, we solve our Hamiltonian by means of NRG, which is a numerically exact technique. In order to use the NRG LJUBLJANA open source code ${ }^{46,47}$, we note that the Hamiltonian in Eq. (1) can be derived as a particular case of a more general, pure fermionic, two-orbital
Anderson model given by

$$
\tilde{H}=\tilde{H}_{i m p}+\tilde{H}_{c}+\tilde{H}_{m i x}
$$

where the impurity Hamiltonian including Coulomb repulsion ${ }^{48}$ reads as follows

$$
\begin{aligned}
\tilde{H}_{i m p}= & \sum_{\alpha} \epsilon_{\alpha} n_{\alpha}+U \sum_{\alpha} n_{\alpha \uparrow} n_{\alpha \downarrow}+U^{\prime} n_{x z} n_{y z}+ \\
& +J_{H} \sum_{\sigma \sigma^{\prime}} h_{x z \sigma}^{\dagger} h_{y z \sigma^{\prime}}^{\dagger} h_{x z \sigma^{\prime}} h_{y z \sigma}+D S_{z}^{2}+ \\
& +J_{H}\left(h_{x z \uparrow}^{\dagger} h_{x z \downarrow}^{\dagger} h_{y z \downarrow} h_{y z \uparrow}+\text { H.c. }\right) \\
= & \sum_{\alpha} \epsilon_{\alpha} n_{\alpha}+U \sum_{\alpha} n_{\alpha \uparrow} n_{\alpha \downarrow}+\left(U^{\prime}-J_{H} / 2\right) n_{x z} n_{y z}+ \\
& -2 J_{H} \vec{S}_{x z} \cdot \vec{S}_{y z}+D S_{z}^{2}+ \\
& +J_{H}\left(h_{x z \uparrow}^{\dagger} h_{x z \downarrow}^{\dagger} h_{y z \downarrow} h_{y z \uparrow}+\text { H.c }\right)
\end{aligned}
$$

being $n_{\alpha}=n_{\alpha \uparrow}+n_{\alpha \downarrow}, n_{\alpha \sigma}=h_{\alpha \sigma}^{\dagger} h_{\alpha \sigma}$ where $\alpha$ indicates the orbital index $\{x z, y z\}$. $U\left(U^{\prime}\right)$ represents the intra(inter-)orbital Coulomb interaction and $J_{H}$ the Hund exchange coupling.

The conduction bands are considered as noninteracting Hamiltonians,

$$
\tilde{H}_{c}=\sum_{k \alpha \sigma} \epsilon_{k \alpha} c_{k \alpha \sigma}^{\dagger} c_{k \alpha \sigma}
$$

and the hybridization term that mixes both contribution is given by

$$
\tilde{H}_{m i x}=\sum_{k \alpha \sigma}\left(V_{\alpha} c_{k \alpha \sigma}^{\dagger} d_{\alpha \sigma}+\text { H.c }\right) .
$$

For simplicity the analysis done below is restricted to $D=0$. The changes for the general case are straightforward. While the Hamiltonian is explicitly invariant under spin rotations, the relation $U^{\prime}=U-2 J_{H}$ comes from spherical symmetry $\mathrm{SO}(3)$ of the Coulomb interaction including orbital degrees of freedom ${ }^{48,49}$.

The diagonalization of $\tilde{H}_{i m p}$ within the two-hole subspace results in the triplet states

$$
\begin{aligned}
& h_{x z \uparrow}^{\dagger} h_{y z \uparrow}^{\dagger}|0\rangle, \\
& \frac{1}{\sqrt{2}}\left(h_{x z \uparrow}^{\dagger} h_{y z \downarrow}^{\dagger}+h_{x z \downarrow}^{\dagger} h_{y z \uparrow}^{\dagger}\right)|0\rangle, \\
& h_{x z \downarrow}^{\dagger} h_{y z \downarrow}^{\dagger}|0\rangle,
\end{aligned}
$$

with energy $E_{T}=E_{2}=\epsilon_{x z}+\epsilon_{y z}+U^{\prime}-J_{H}$, together with the following two singlets, degenerate as a consequence of the $S O(3)$ symmetry of the interaction,

$$
\begin{aligned}
& \frac{1}{\sqrt{2}}\left(h_{x z \uparrow}^{\dagger} h_{y z \downarrow}^{\dagger}-h_{x z \downarrow}^{\dagger} h_{y z \uparrow}^{\dagger}\right)|0\rangle, \\
& \frac{1}{\sqrt{2}}\left(h_{x z \uparrow}^{\dagger} h_{x z \downarrow}^{\dagger}-h_{y z \uparrow}^{\dagger} h_{y z \downarrow}^{\dagger}\right)|0\rangle,
\end{aligned}
$$


with energy $E_{S_{\text {low }}}=\epsilon_{x z}+\epsilon_{y z}+U^{\prime}+J_{H}$, and finally one excited singlet,

$$
\frac{1}{\sqrt{2}}\left(h_{x z \uparrow}^{\dagger} h_{x z \downarrow}^{\dagger}+h_{y z \uparrow}^{\dagger} h_{y z \downarrow}^{\dagger}\right)|0\rangle
$$

with energy $E_{S_{e x}}=\epsilon_{x z}+\epsilon_{y z}+U+J_{H}$.

The two-orbital model of Eq. (2), neglecting the pairhopping term [the last one in Eq. (3)], has been studied by using $\mathrm{NRG}$ in the context of impurity ${ }^{50-55}$ and lattice models within the dynamical mean field theory ${ }^{56}$. Specifically, the work of Nishikawa and Hewson ${ }^{55}$ focuses on the role of Hund's interaction and as we shall see, some of our results agree with theirs.

In any case, since we are interested in retaining only the lowest triplet state in the configuration with two particles, we can take $U^{\prime}=J_{H}$ with $J_{H}$ and $U$ large enough so that the singlets play no role and can be removed from the impurity Hilbert space. The condition $U^{\prime}=U-2 J_{H}$ is not satisfied but this only breaks the symmetry of irrelevant high-energy singlet states. We also neglect, as in previous works, the pair-hopping term (the double occupied states with both holes in the same orbital are excluded by large $U)$. Then, the surviving two-particle states belong to the triplet with energy $E_{2}=E_{T}=\epsilon_{x z}+\epsilon_{y z}$, and the other relevant configuration has two one-particle doublets with energy $E_{1}=\epsilon_{\alpha}$. Note that the zero-particle state has a finite energy $E_{0}=0$, but higher than the one-particle state energies; consequently, it can also be discarded in the study of the low energy physics of the $S=1$ impurity.

The resulting accessible Hilbert space contains only the three components of the two-particle spin $S=1$ and the two one-particle doublets and, therefore, the Hamiltonian mixes these two configurations in identical form that the corresponding one in Eq. (1). These assumptions highly simplify the use of NRG.

As mentioned in the introduction, we present calculations in the strong Hund's coupling limit, in which a $S=1$ ground state is screened by spin- $1 / 2$ electrons. Furthermore, the role of the anisotropy term, not included in previous studies, $D S_{z}^{2}$ is considered. We note that Ref. 56 analyzes some aspects of this anisotropy contribution.

\section{B. Non Crossing Approximation}

Although the non-crossing approximation ${ }^{57}$ for fully screened models fails to accurately reproduce Fermi liquid relationships at zero temperature, it gives accurate results at finite and high excitation energies. For instance, the intensity and the width of the charge-transfer peaks of the spectral density (those which correspond to differences in energy between neighboring configurations, such as the dot level $\epsilon$ and $\epsilon+U$ in the simplest one-channel SU(2) impurity Anderson model) given by NCA were found ${ }^{58,59}$ to be in agreement with other theoretical methods ${ }^{60,61}$ and also with experiments in which a marked asymmetry in the intensity and width of the resonances for bias voltage $V \neq 0$ was observed, depending on the polarity of $V^{62}$. Furthermore, it has a natural extension to non-equilibrium conditions ${ }^{63}$ and it is especially suitable for describing satellite peaks away from the zero bias voltage $26,28,64$. In addition, the NCA Haldane shift ${ }^{65}$ (the renormalization of the bare $\epsilon_{\alpha}$ energy due to many body correlations) was found to be in agreement with the Haldane's prediction ${ }^{58,59,64}$. We remind the reader that the charge-transfer peaks as any other satellite peak in the spectral function are artificially broadened within NRG due to the logarithmic discretization of the conducting band ${ }^{66}$ and hence the NCA solution, which is free of this shortcoming became a useful alternative treatment. For the $(S=1 / 2)$ one-channel case, the NCA reproduces well the scaling relations with temperature $T$ and bias voltage in the Kondo regime ${ }^{67}$.

Within the NCA framework, we rewrite the Hamiltonian in Eq. (1) by using a pseudo-particle representation of the Hubbard operators $\left|M_{i}><M_{j}\right| \longrightarrow \hat{a}_{M_{i}}^{\dagger} \hat{a}_{M_{j}}$, which renders the model to the following form

$$
\begin{aligned}
H^{\prime} & =\sum_{M_{2}}\left(E_{2}+D M_{2}^{2}\right) \hat{a}_{M_{2}}^{\dagger} \hat{a}_{M_{2}}+\sum_{\alpha M_{1}} E_{\alpha} \hat{a}_{\alpha M_{1}}^{\dagger} \hat{a}_{\alpha M_{1}}+ \\
& +\sum_{\nu k \alpha \sigma} \epsilon_{\nu k} c_{\nu k \alpha \sigma}^{\dagger} c_{\nu k \alpha \sigma}+ \\
& +\sum_{M_{1} M_{2}} \sum_{\alpha \nu k \sigma} V_{\nu \alpha}\left\langle 1 M_{2} \mid \frac{1}{2} \frac{1}{2} M_{1} \sigma\right\rangle\left(\hat{a}_{M_{2}}^{\dagger} \hat{a}_{\alpha M_{1}} c_{\nu k \alpha \sigma}+\text { H.c. }\right)
\end{aligned}
$$

In addition, the number of pseudo-particles should satisfy the constraint

$$
\sum_{M_{2}} \hat{a}_{M_{2}}^{\dagger} \hat{a}_{M_{2}}+\sum_{\alpha M_{1}} \hat{a}_{\alpha M_{1}}^{\dagger} \hat{a}_{\alpha M_{1}}=1 .
$$

The approximation makes use of an individual dynamics of each class of particles, which obeys the following self-consistent equations for the corresponding selfenergies

$$
\begin{aligned}
\Sigma_{\alpha}(\omega) & =\frac{1}{\pi} \int d \epsilon f(\epsilon) \Delta_{\bar{\alpha}}(\epsilon)\left[G_{21}(\epsilon+\omega)+\frac{1}{2} G_{20}(\epsilon+\omega)\right] \\
\Sigma_{2}(\omega) & =\frac{1}{\pi} \int d \epsilon f(-\epsilon) \sum_{\alpha} \Delta_{\alpha}(\epsilon) G_{\bar{\alpha}}(\omega-\epsilon)
\end{aligned}
$$

where $\Delta_{\alpha}(\epsilon)=\pi V_{\alpha}^{2} \rho_{\alpha}^{(c)}(\epsilon)$ represents the hybridization of the impurity with the $\alpha$-channel of conduction electron of density $\rho_{\alpha}^{(c)}(\epsilon)$. The retarded Green function $G_{21}\left(G_{20}\right)$ takes into account the $\pm 1(0)$ components of the triplet, while $G_{\alpha}$ stands for the doublet of symmetry $\alpha$. The temperature is included within the Fermi function $f(\epsilon)$.

Details of the technique and its numerical evaluation can be found in the above mentioned references.

\section{Numerical results}

For a numerical resolution of the model at hands, constant and symmetric unperturbed conduction bands, $\rho_{\alpha}^{(c)}$, 
in the range $[-W, W]$ are considered. Furthermore, without loss of generality (except for the magnitude of the current) we assume symmetric coupling to the leads, $V_{\alpha L}=V_{\alpha R}=V_{\alpha} / \sqrt{2}$, independent of energy, which implies a constant resonant-level width $\Delta_{\alpha}=\pi V_{\alpha}^{2} \rho_{\alpha}^{(c)}$. We also define $\Delta=\Delta_{x z}+\Delta_{y z}$

We restrict ourselves to the case in which both conducting band densities $\rho_{\alpha}^{(c)}$, hybridization hoppings, $V_{\alpha}$, and the impurity levels $\epsilon_{\alpha}$ are degenerate by symmetry, inspired in the real situation of the Ni impurity in an $\mathrm{Au}$ chain (along the z-axis) for which the relations $\rho_{5 d_{x z}}^{(c)}(\omega)=\rho_{5 d_{y z}}^{(c)}(\omega), V_{x z}=V_{y z}$, and $\epsilon_{x z}=\epsilon_{y z}$ hold.

We define the unit of energy $(W=1)$ such that $\Delta=0.01$. We also take $U^{\prime}=J_{H}=1000$ in the rest of the paper, unless otherwise stated. This choice displaces to very high energy all excited states of the $d^{8}$ configuration, leaving only the triplet states $\left|1 M_{2}\right\rangle$ at low energies. As explained in Section II A, this choice allows us to represent the model Eq. (1) in a form suitable for the NRG code. Except for the subsection III E, in the rest of the paper we take an anisotropy $D=0$.

For the cases in which the system is in the Kondo regime, that is for $\Delta$ much smaller than the other bare energy scales, we define the Kondo temperature $T_{K}$ from the equilibrium conductance in the following way, $G\left(T_{K}\right)=G(T \rightarrow 0) / 2$. This definition of $T_{K}$ gives values of the same order of magnitude than the corresponding one obtained from the half width at half maximum of the Kondo resonance in the spectral density ${ }^{68}$. Regarding the bare orbital energies, we employ the notation $\epsilon_{d}=E_{2}-E_{1}$.

\section{A. Entropy and occupancy}

We start our discussion of the numerical results of the model of Eq. (1) by analyzing the NRG results for the impurity contribution $S_{i m p}(T)$ to the total entropy as a function of temperature. The top panel of Fig. 2 shows the calculated $S_{i m p}(T)$ for four different set of parameters, $\epsilon_{d}=\{-4,-3.5,-3,-2.5,-2\} \Delta$ as a function of temperature. The case $\epsilon_{d}=-2 \Delta$ correspond to the $a b$ initio parameters for the $\mathrm{Ni}$ impurity in an O-doped $\mathrm{Au}$ chain $^{42}$ (black solid line).

At large enough temperatures, $e^{S_{i m p}}$ saturates at the value imposed by the dimension of the local Hilbert space $g=7$ given by the three components of the triplet and the 4-fold degenerate states corresponding to the two doublets. As the temperature is lowered, an intermediate plateau can be observed in which $e^{S_{i m p}} \simeq 3$, due to the triplet. This is the local-moment regime characterized by the fact that the charge fluctuations are frozen. It corresponds to the Kondo limit of the model, obtained from a Schrieffer-Wolff transformation in Ref. 42, in which only spin fluctuations are present.

As expected for the symmetry of the model, in which two spin- $1 / 2$ conduction bands coherently screen the to-
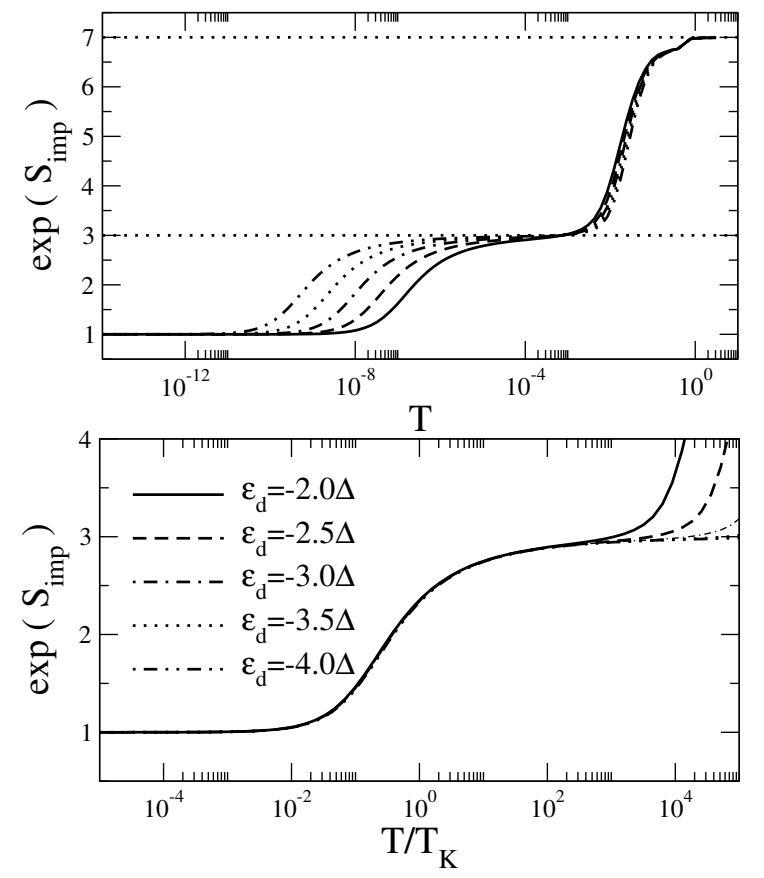

FIG. 2: Top panel: NRG impurity contribution to the entropy as a function of temperature, $S_{i m p}(T)$, for the model of Eq. (2). Lower panel: Same data in units of $T / T_{K}$, being $T_{K}=6.7 \times 10^{-7}, 1.7 \times 10^{-7}, 4.0 \times 10^{-8}, 9.2 \times 10^{-9}, 2.1 \times 10^{-9}$ for $-\epsilon_{d} / \Delta=2,2.5,3,3.5,4$, respectively.

tal impurity spin $S=1$, when the temperature falls under $T_{K}$, the system enters the strong-coupling regime, and the value of $e^{S_{i m p}}$ tends to one, corresponding to the Fermi-liquid non-degenerate Kondo ground state.

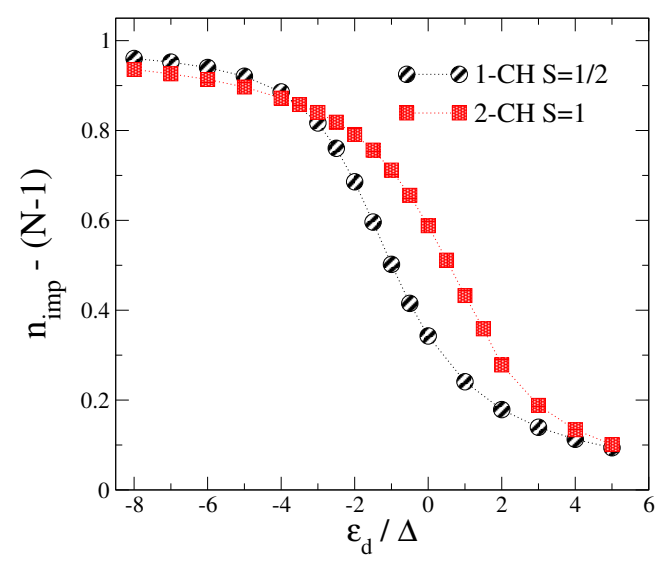

FIG. 3: (Color online) NRG total impurity occupancy, $n_{i m p}-$ $(M-1)$, as a function of $\epsilon_{d}$, being $M$ the number of channels. Squares indicate the results for the model of Eq. (2), $M=2$. The circles correspond to the impurity population for the spin $s=1 / 2$ infinite $U$-limit one-channel Anderson model where $M=1$.

In the lower panel of Fig. 2 we show the same data but with temperatures scaled by the corresponding Kondo ones. It is clear that, within the local-moment and 
strong-coupling regimes, the system displays universality and all curves have the same temperature dependence when the latter is expressed in units of $T_{K}$, being this the only relevant scale of the model.

Note that, for the ab-initio parameters, there is also an extended range of temperatures, of about 7 decades, $10^{-5} \lesssim T / T_{K} \lesssim 10^{2}$, in which $e^{S_{i m p}}$ falls on top of the others corresponding for more negative values of $\epsilon_{d}$. This is a common feature of the regime of parameters $\epsilon_{d} \ll-\Delta$ for which the Kondo model is valid. However, $\epsilon_{d}=-2 \Delta$ would seem not negative enough to suppress charge fluctuations. Indeed, the relation $\epsilon_{d}=-2 \Delta$ for the case of the well studied one-channel spin-1/2 Anderson impurity characterizes the mixed valence regime of the model ${ }^{67}$. To clarify this point, we compared the NRG results for the total impurity occupancy $n_{i m p}$ as a function of the energy $\epsilon_{d}$ in the present case of the 2-channel $S=1$ model and the one corresponding to the one-channel spin-1/2 impurity model. The result is shown in Fig. 3.

Remarkably, the mixed valence regime in the case of the 2-channel $S=1$ is strongly suppressed in the range of negative values of $\epsilon_{d}$. In fact, when $-\epsilon_{d} / \Delta=1$ the impurity is near $70 \%$ occupied as compared with near $50 \%$ in the case of the ordinary one-channel spin-1/2 model. From this result, we conclude that the realistic parameters representing the $\mathrm{Ni}$ impurity in the $\mathrm{Au}$ chain correspond to a description of the system within its Kondo regime. This point will be discussed further in the following subsections.

\section{B. Spectral density}

In Fig. 4 we show the NRG impurity spectral density per channel and per spin $\rho_{\alpha \sigma}$ as a function of the frequency for several values of the bare energy level $\epsilon_{d}$ and at sufficiently low temperature as compared with the Kondo one for each $\epsilon_{d}$. The resulting spectral function is quite similar to the corresponding one in the case of the one-channel infinite $U$-limit spin-1/2 Anderson impurity. Indeed, there is only one charge transfer peak located near the bare energy $\epsilon_{d}=E_{2}-E_{1}$ which indicates the energy needed to put a second hole (-electron) in the impurity to form one of the triplet states. Furthermore, the narrow Kondo peak at the Fermi level has a width of the order of $T_{K}$ (visible in the inset of the figure) and its intensity is imposed by the usual Friedel sum rule, $\rho_{\alpha \sigma}(0)=\frac{1}{\pi \Delta} \sin ^{2}\left(\frac{\pi n_{\alpha}}{2}\right)$, where $n_{\alpha}$ is the total population of the $\alpha$-level.

However, there are non-trivial differences between the spectral density of the present model and one-channel infinite $U$-limit spin-1/2. The first one is related to the reduced width of the Kondo resonance, whose discussion will be given in detail in the next subsection. Here we focus on the manifestation of the many-body interactions in the charge-transfer peak. Specifically, we examine its width and position. To this purpose,

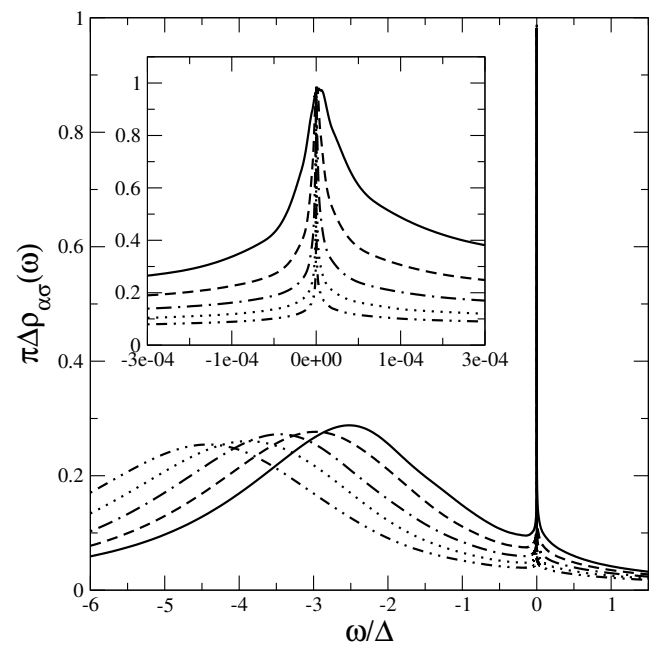

FIG. 4: NRG impurity spectral density as a function of frequency for the same set of values of $\epsilon_{d}$ and other parameters as in Fig. 2.

we employ the NCA results for the spectral density. Although the NCA does not provide accurate results for the low energy physics of the model at hands, in particular $T_{K}$ is found to be overestimated as we will show in the appendix A, it is especially suitable for the study of the charge transfer peak. We remind the reader that the logarithmic discretization of the conduction band produces an artificial broadening of the charge transfer peaks within the NRG procedure ${ }^{66}$. Therefore, we use NCA when analyzing high energy scales.

Position and width of the charge transfer peak. In Fig. 5 we present the NCA results for the spectral density for the same set of parameters as in Fig. 4.

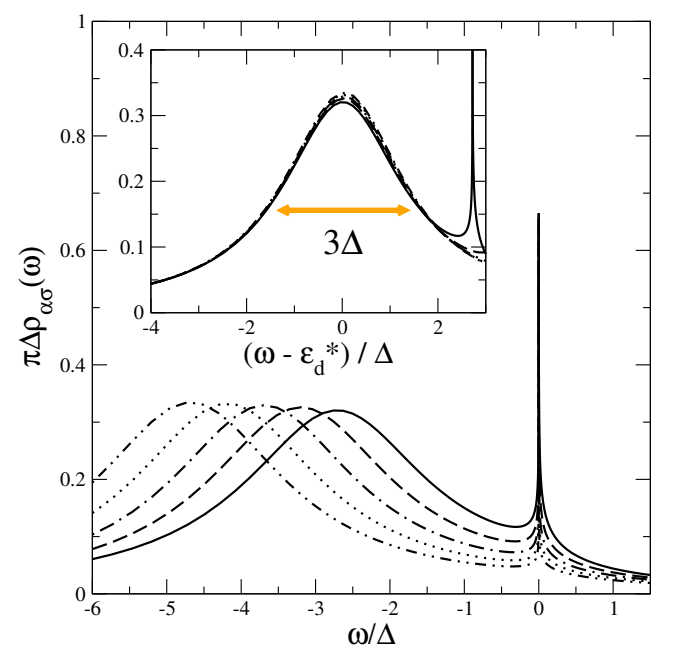

FIG. 5: Same as Fig. 4 calculated with NCA. The inset shows the charge-transfer peaks shifted by $\epsilon_{d}^{*}$ (see Eq. 13). 
Renormalization of the bare energy $\epsilon_{d}$ : The Haldane shift. By means of scaling theory, Haldane ${ }^{65}$ has showed that for the asymmetric spin $s=1 / 2$ one-channel Anderson model $\left(U \gg\left|\epsilon_{d}\right|, \Delta\right)$, the renormalized value of the level $\epsilon_{d}$ is given by $\epsilon_{d}^{*}=\epsilon_{d}+(\Delta / \pi) \ln (W / \Delta)$. As we have mentioned in section (IIB), the NCA has proved to be capable of providing accurate results for such energy shift ${ }^{58,59,64}$. The sign and the main features of the dependence with $\Delta$ of the Haldane shift can be understood by a simple argument. Due to the hybridization with the conduction band, the bare energies of the empty and the single occupied states are renormalized. While the empty state is mixed with both single occupied states and, consequently, its energy is lowered by an amount proportional to $2 \Delta$, a single occupied state can only be mixed with the empty state, so its bare energy $\epsilon_{d}$ is reduced an amount $\propto \Delta$. As $\epsilon_{d}^{*}$ is the energy difference between both renormalized energies, we have $\Delta \epsilon \equiv \epsilon_{d}^{*}-\epsilon_{d} \propto \Delta$. Of course, the logarithmic term in $\Delta \epsilon=(\Delta / \pi) \ln (W / \Delta)$ can only be obtained through the scaling process. This approach is easy to generalize to the $\mathrm{SU}(N)$ impurity Anderson model for valence fluctuations between the configurations with zero and one localized particles ${ }^{59}$.

In the case of the model describing a $\mathrm{Ni}$ impurity and following Haldane's approach, starting from the triplet state $|11\rangle=h_{x z \uparrow}^{\dagger} h_{y z \uparrow}^{\dagger}|0\rangle$ there are two different processes with hybridization $V$ that connect this state with one containing only one hole, implying that its renormalized energy goes down an amount $\propto 2 \Delta$. On the other hand, starting from a given state $|\alpha \uparrow\rangle$ and assuming $J_{H} \rightarrow \infty$ there are also two processes, one to the state |11) with hybridization $V$ and other one to the state $|10\rangle=\frac{1}{\sqrt{2}}\left(h_{x z \uparrow}^{\dagger} h_{y z \downarrow}^{\dagger}+h_{x z \downarrow}^{\dagger} h_{y z \uparrow}^{\dagger}\right)|0\rangle$ with hybridization $V / \sqrt{2}$, in such a way that the bare energy of the onehole state goes down $\propto 3 \Delta / 2$. This energy gain is lower than that corresponding to the triplet states, and so we expect $\Delta \epsilon \propto-\Delta / 2$. In fact, using scaling arguments similar to those of Ref. 65 , the renormalized energy $\epsilon_{d}^{*}$ becomes

$$
\epsilon_{d}^{*}=\epsilon_{d}-(\Delta / 2 \pi) \ln (W / \Delta) .
$$

Note that in comparison to the known one-channel $s=$ $1 / 2$ case, the shift $\Delta \epsilon$ has the opposite sign and its magnitude is reduced by a factor $1 / 2$.

We calculate $\epsilon_{d}^{*}$ as the energy position of the maximum in the charge-transfer peak of the spectral density in both, NRG and NCA. Fig. 5 explicitly shows the shift towards negative energies. In Fig. 6 the values of $\epsilon_{d}^{*}$ are shown as a function of $\epsilon_{d}$ in units of $\Delta$. Both techniques display a linear behavior, however with NCA we obtain a remarkably better well defined linear function (correlation coefficient 0.999 ) with a slope close to 1 and a constant of -0.722 in agreement with the second term of the r.h.s. of Eq. 13, -0.733 .

Width of the charge-transfer peak. The inset of Fig. 5 shows a detail of the charge-transfer peak shifted by $\epsilon_{d}^{*}$.

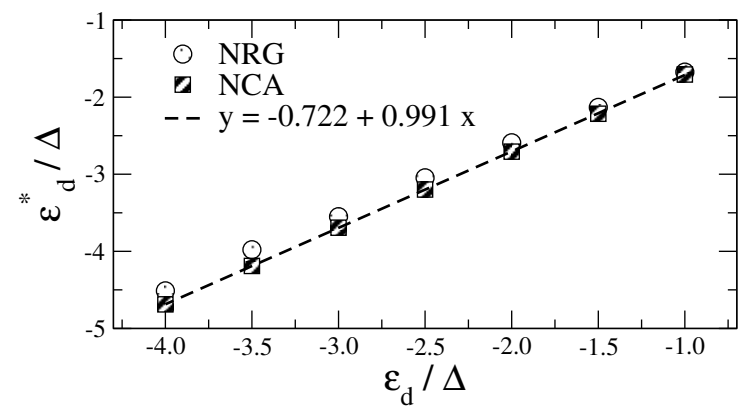

FIG. 6: Renormalized position of the charge-transfer peak, $\epsilon_{d}^{*}$, as a function of $\epsilon_{d}$. Other parameters as in Fig. 2.

We remind the reader that the half-width of this peak in the case of the one-channel $s=1 / 2$ case is found to be $2 \Delta$, where $\Delta$ corresponds to the one-body broadening already present in the non-interacting model ${ }^{60}$. The prefactor which in general is $N$ for the $\mathrm{SU}(N)$ case ${ }^{59}$ has its origin in effects of the interaction.

In the present case the half-width of the charge-transfer peak is $3 \Delta / 2$. We trace back this difference with the following qualitative argument: the half-width $2 \Delta$ in the one-channel $s=1 / 2$ model reflects the two processes by which the excited empty state is connected to the single-occupied ground state with hybridization $V^{59}$. On the other hand, for the $s=1$ model, the excited onehole states are connected to the $S_{z}=1$ (or $S_{z}=-1$ ) component of the ground state with hybridization $V$, and to the $S_{z}=0$ component with hybridization $V / \sqrt{2}$. As a consequence, the half-width is now $3 \Delta / 2$.

\section{Kondo temperature and Kondo resonance narrowing effect}

The Kondo temperature is undoubtedly the most relevant energy scale in the Kondo phenomena simply because it represents a universal scale, in terms of which all physical properties follow a given dependence as a function of $T / T_{K}$ without being affected by the other parameters of the model. Therefore, it is always desirable to have an analytical expression for such a scale.

Previous studies on the basis of NRG calculations have found that the introduction of Hund's coupling into the Anderson model causes an exponential reduction in the Kondo temperature ${ }^{55,56}$. Particularly, our model assumes an infinite Hund's coupling and belongs to the same class as the one studied by Nevidomskyy and Coleman in Ref. 38 by means of scaling arguments. Applied to our case, their main result was the existence of a factor $1 / 2$ in the exponent of the expression of $T_{K}$ for the full screened spin $S=1$ model in comparison with the corresponding one for the spin $s=1 / 2$. In general, all approaches agree in the exponential dependence of the Kondo scale, given by $\exp \left(\frac{\pi \epsilon_{d}}{\Delta}\right)$ instead of $\exp \left(\frac{\pi \epsilon_{d}}{2 \Delta}\right)$ of the usual one-channel spin $s=1 / 2$ case. 
Therefore, a relation of the form $T_{K}^{S=1} \sim\left(T_{K}^{s=1 / 2}\right)^{2}$ is expected being $T_{K}^{s=1 / 2}=\sqrt{W \Delta} \exp \left(\frac{\pi \epsilon_{d}}{2 \Delta}\right)$. Note that the latter can be obtained from the Haldane ${ }^{65}$ expression $T_{K}^{s=1 / 2}=\Delta \exp \left(\frac{\pi \epsilon_{d}^{*}}{2 \Delta}\right)$ using $\epsilon_{d}^{*}=\epsilon_{d}+(\Delta / \pi) \ln (W / \Delta)$. Following similar arguments, using $T_{K}^{S=1}=\Delta \exp \left(\frac{\pi \epsilon_{d}^{*}}{\Delta}\right)$ and the renormalized level position given by Eq. (13) we obtain

$$
T_{K}^{S=1}=c \sqrt{\Delta / W^{3}}\left(T_{K}^{s=1 / 2}\right)^{2}=c \sqrt{\Delta^{3} / W} e^{\frac{\pi \epsilon_{d}}{\Delta}},
$$

being $c$ a constant of the order of one.
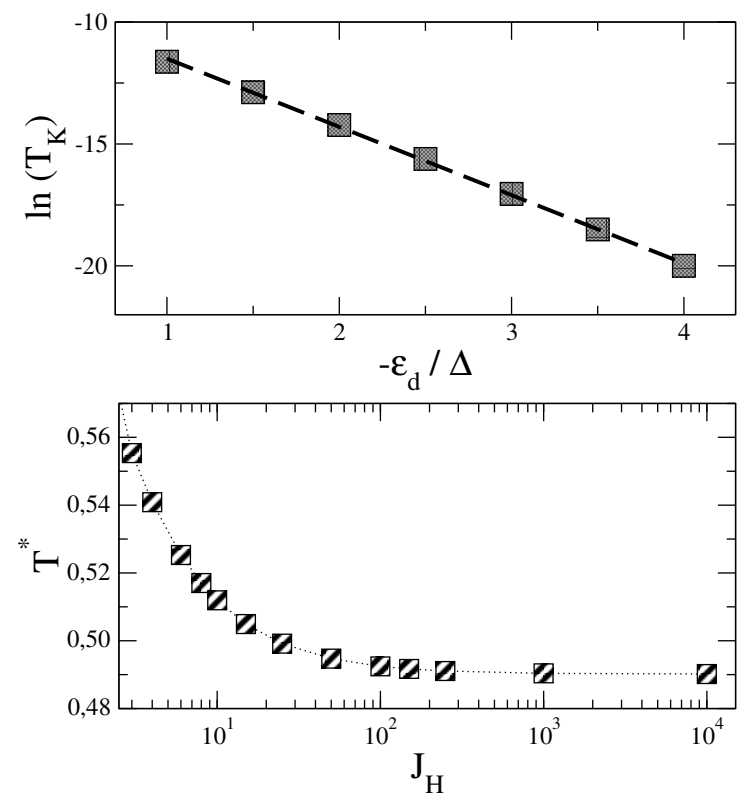

FIG. 7: Top panel: The squares indicate the NRG Kondo temperature as a function of $\left|\epsilon_{d}\right| / \Delta$ calculated for $\left|J_{H}\right|=$ $1000 W$. Dashed line: linear fitting giving $\ln \left(T_{K}\right)=-8.70-$ $2.80\left|\epsilon_{d}\right| / \Delta$ with a correlation coefficient of 0.999 . Lower panel: $T^{*}=T_{K}\left(J_{H}\right) / \sqrt{\Delta / W^{3}}\left(T_{K}^{s=1 / 2}\right)^{2}$ as a function of $J_{H}$ for $\left|\epsilon_{d}\right| / \Delta=3$.

Our numerical NRG data for the Kondo temperature in case of large enough $J_{H}$, in such a way that the local moments become locked into a spin $S=1$, confirm this exponential dependence. In the top panel of Fig. (7) we show $T_{K}$ (obtained from the conductance as described at the beginning of this Section) as a function of $\left|\epsilon_{d}\right| / \Delta$, together a linear fit of the data which exhibits a slope that differs from the factor $\pi$ in less than $10 \%$.

Regarding the dependence of $T_{K}$ with $J_{H}$, in the lower panel of Fig. (7) we show the calculated Kondo temperature in units of $\sqrt{\Delta / W^{3}}\left(T_{K}^{s=1 / 2}\right)^{2}$ for a fixed value of $\left|\epsilon_{d}\right| / \Delta=3$. It can be observed that the relation in Eq. (14) is satisfied in the asymptotic behavior $J_{H} \rightarrow \infty$, being the constant $c=0.49 \sim 1 / 2$. In fact, we have verified that this constant varies between 0.5 to 1.0 for the whole set of values of $\left|\epsilon_{d}\right| / \Delta$ presented in top panel of Fig. (7).

\section{Electrical conductance}

Since the ground state of model Eq. (1) for $D=0$ is a Fermi liquid, transport measurements through the proposed nanowire should exhibit universal behavior at low enough temperatures, for parameters that drive the system inside the Kondo regime. However, as we have shown in previous sections, the universal dependence of the observables, for instance as a function of temperature, is expected to be different from the well known spin $s=1 / 2$ case. Here, we analyze the NRG results for the equilibrium electrical conductance, $G(T)$. The temperature dependence of the conductance through the $\mathrm{Ni}$ atom depends on the total impurity spectral function $\rho(\omega)=\sum_{\alpha \sigma} \rho_{\alpha \sigma}(\omega)$, and it can be calculated from the following expression ${ }^{63}$

$$
G(T)=G_{0} \frac{\pi \Delta}{2} \int d \omega\left(-f^{\prime}(\omega)\right) \rho(\omega),
$$

where $G_{0}=2 e^{2} / h$ is the quantum of conductance. Note that from the expected Friedel sum rule at zero temperature, $\sum_{\alpha \sigma} \rho_{\alpha \sigma}(0) \sim \frac{4}{\pi \Delta}$, the maximum value of the conductance should be $G(T \rightarrow 0)=2 G_{0}$, twice of the usual one channel case. We remind the reader that this is not always the case in two-channel models. For instance, in the overscreened $s=1 / 2$ two-channel case, the maximum value of the total conductance is found to be $G_{0} / 2^{25,26}$. The present result is a consequence of the Fermi liquid nature of the ground state, due to the full screening of the impurity spin.

In the top panel of Fig. 8, we show the NRG results for $G(T)$ that correspond to the model of Eq. (2) for several values of $\epsilon_{d}$. The lower panel displays the same data as in the top panel with the temperature scaled by the corresponding Kondo one. As expected, for temperatures $T \lesssim T_{K}$ the whole set of curves follows the same dependence in units of $T / T_{K}$, which confirm that $T_{K}$ is the only one relevant energy scale of the model within the Kondo regime. Furthermore, the case $-\epsilon_{d} / \Delta=2$ also follows the universal dependence reinforcing the conclusion that, for the parameters representing the Ni impurity in the O-doped Au chain, the system lies within the Kondo regime.

Regarding the dependence of $G$ with $T / T_{K}$, it is well known that the empirical expression

$$
G(T)=\frac{G_{s}}{\left[1+\left(2^{1 / s}-1\right)\left(T / T_{K}\right)^{2}\right]^{s}},
$$

with $s=0.22$ and $G_{s}$ the conductance at temperature $T=0$, matches not only experimental results but also NRG calculations in the case of the spin $s=1 / 2$ onechannel Anderson model ${ }^{3,69}$. A similar scaling law has 

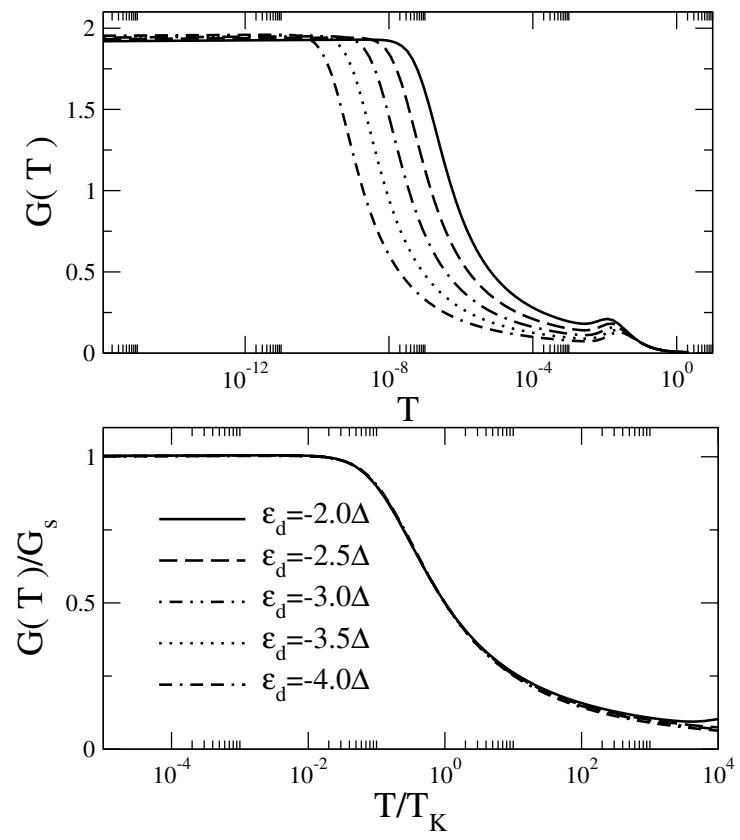

FIG. 8: Top panel: Equilibrium conductance $G(T)$ in units of $2 e^{2} / h$ as a function of temperature. Lower panel: The same data that in the top panel in units of $T / T_{K}$ (see caption of Fig. 2 for the values of $T_{K}$ ) and normalized by the saturated conductance $G_{s}=G(T \rightarrow 0)$

been used to fit experimental data and one-channel NRG results for the resistivity due to magnetic impurities with spin $S=1 / 2,1$ and $3 / 2$ in $\mathrm{Fe}$ and $\mathrm{Ag}$ by Costi et $a l^{70}$. Although the scaling function in this case is different from that of $G(T)$ both coincide for $T \ll T_{K}{ }^{71}$. Similar scaling functions were used to fit $G(T)$ for the underscreened Kondo model ${ }^{18,72}$. Here, we show that also for our model the scaling is noticeable different that in the $s=1 / 2$ case, in spite of being a fully compensated Kondo effect.

Fig. (9) displays the NRG results for the total conductance per channel, $G_{\alpha}(T)=\sum_{\sigma} G_{\alpha \sigma}(T)$, in units of its maximum $G_{s}$ as a function of $T / T_{K}$ for a selected value of $\epsilon_{d}$ well inside the Kondo regime. The red solid line indicates the temperature dependence given by Eq. (16) for the spin $s=1 / 2$ model. As it can be seen, the numerical data corresponding to the full screening of the spin $S=1$ appreciably deviates from the latter.

Our results indicate that the relation given in Eq. (16) is still valid in the present case but with a different factor $s=0.15$. We have verified that similar coefficients, $0.15<s<0.17$, appear when the formula is applied to other, negative enough, values of $\epsilon_{d}$. Curiously, a similar exponent $s=0.16$ was found for the fit of the resistivity due to $S=1$ Kondo impurities in the underscreened case. Empirical formulas like (16) have been shown to be a very useful tool in order to discern the spin of impurities in experimental underscreened Kondo systems ${ }^{18,72}$, and so, the good agreement between the NRG $G(T)$ and Eq. (16), would allow to identify $S=1$ fully screened

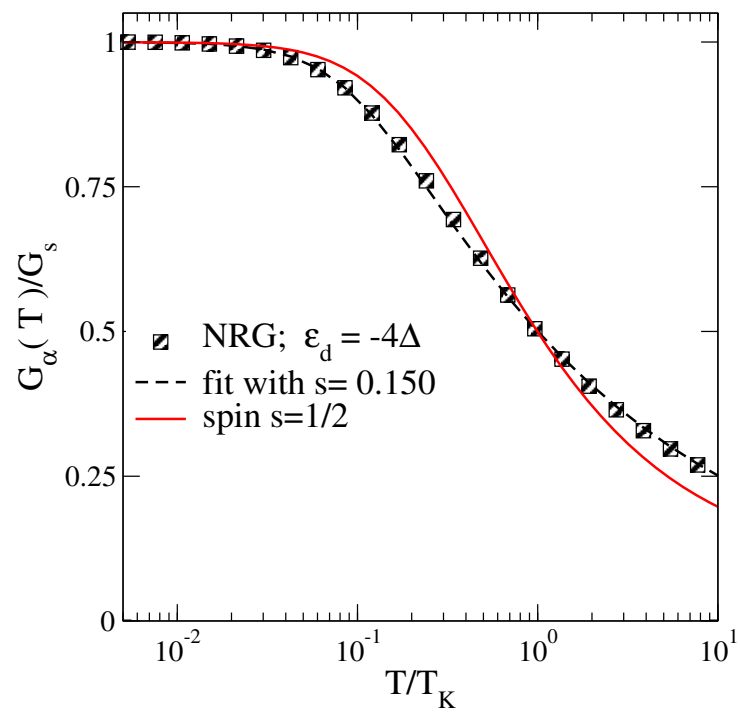

FIG. 9: (Color online) Squares: NRG data for $G_{\alpha}(T)=$ $\sum_{\sigma} G_{\alpha \sigma}(T)$ in units of its maximum $G_{s}$ as a function of $T / T_{K}$, for $\epsilon_{d}=-4 \Delta$. Dashed black line: fitting of numerical data with expression Eq. (16) with $s=0.15$. Solid red line: Eq. (16) with $s=0.22$.

systems.

\section{E. Role of the single-ion magnetic anisotropy}

A key ingredient in magnetic nanosystems is the presence of rather large single-ion magnetic anisotropies $D S_{z}^{2}$, due to the enhanced spin-orbit coupling brought about by the lower symmetries than in bulk systems ${ }^{73-77}$. In fact, cluster and $a b$-initio calculations ${ }^{42}$ yields an appreciable positive $D \sim 8.5 \mathrm{meV}$ for the Ni atom embedded into the O-doped $\mathrm{Au}$ chains.

Very recently, working with the same model of Eq. (2), we have uncovered a topological quantum phase transition between two Fermi liquids as a function of the magnetic anisotropy ${ }^{43}$. For $D<D_{c} \simeq(2-3) T_{K}^{0}$ ( $T_{K}^{0}$ is the Kondo temperature for $D=0$ ), the impurity is fully Kondo screened as in the $D=0$ case, with a Kondo temperature that, surprisingly, scales as a power law of $D$,

$$
T_{K}(D) \propto T_{K}^{0}\left(\frac{D_{c}-D}{D_{c}}\right)^{2} .
$$

close to $D_{c}{ }^{43}$. On the hand, for $D>D_{c}$ the impurity spin is quenched by the anisotropy, giving rise to a topological non-trivial Fermi liquid ground state, with a non-zero Luttinger integral $I_{L}{ }^{43,78}$. Due to this fact the system cannot be adiabatically connected to a non-interacting one. Therefore we have named it a non-Landau Fermi liquid $^{43}$. At the critical point, the system exhibits several non-Fermi liquid signatures of the two-channel $s=1 / 2$ Kondo (2CK) effect. 
We remind the reader that in a Fermi liquid, as in a simple pure metal, the life time of the quasiparticles for small excitation energy $\omega$ from the Fermi energy, scales as $\omega^{-2}$ at zero temperatures. Landau postulated that due to restrictions of phase space imposed by Pauli principle, the same behavior should take place in interacting systems. However for some strongly interacting systems, like the overscreened Kondo models, this picture breaks down and the quasiparticles have finite lifetime even at $\omega=0$. An intermediate case are the marginal Fermi liquids (corresponding to underscreened Kondo models) in which the lifetime is infinite at $\omega=0$ but has a non analytical dependence on $\omega^{22,29}$ In our case for $D \neq D_{c}$, the system is a Fermi liquid. Until recently, the natural expectation was that a Fermi liquid, like a non-interacting system was characterized by $I_{L}=0$, but as shown first by Curtin et al. ${ }^{78}$, some interacting system (such as ours for $D>D_{c}$ ) behave as Fermi liquids with $I_{L} \neq 0$. $I_{L}$ has a topological character and can only have discrete values $^{43,79}$.

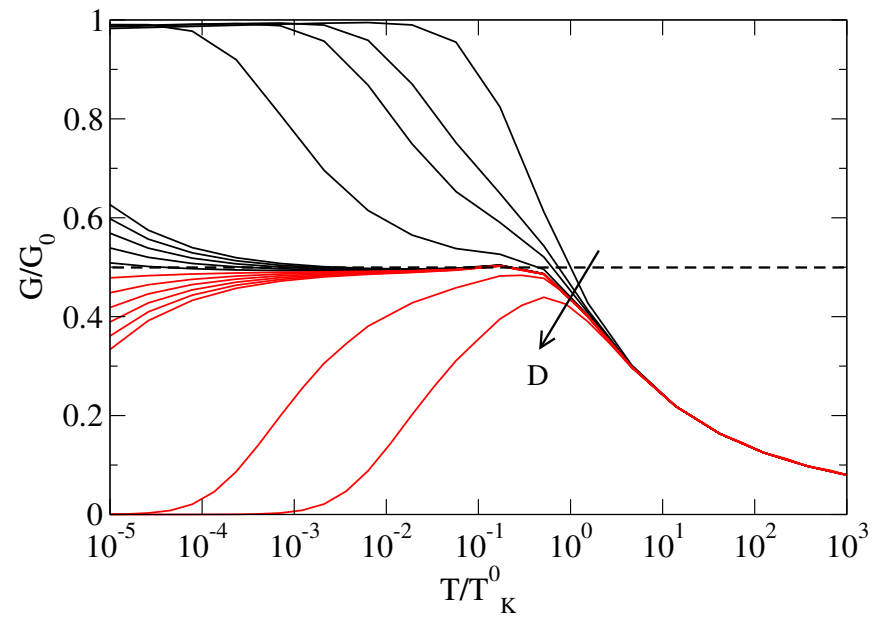

FIG. 10: (Color online) NRG conductance $G_{\alpha}(T) / G_{0}$ of the $S=1$ Kondo impurity model for several positive magnetic anisotropies $D$ across the topological quantum phase transition. The black (red) curves correspond to $D<D_{c}\left(D>D_{c}\right)$. From top to bottom: $D=0.0,1 \times 10^{-4}, 1.15 \times 10^{-4}, 1.3 \times 10^{-4}$, $1.35 \times 10^{-4}, 1.3505 \times 10^{-4}, 1.351 \times 10^{-4}, 1.315 \times 10^{-4}$, $1.352 \times 10^{-4}, 1.3525 \times 10^{-4}, 1.353 \times 10^{-4}, 1.3535 \times 10^{-4}$, $1.354 \times 10^{-4}, 1.345 \times 10^{-4}, 1.355 \times 10^{-4}, 1.4 \times 10^{-4}, 1.6 \times 10^{-4}$. The parameters are $J=0.2$ and $W=1$. We take $\Lambda=3$ and keep $4000 \mathrm{NRG}$ states. For these parameters, $T_{K}^{0}=4.2 \times 10^{-5}$ and $D_{c}=1.352 \times 10^{-4}$.

To illustrate the generic appearance of this topological quantum phase transition for the $S=1$ impurity, we consider the Kondo limit of the two-orbital Anderson model (2), that is, a $S=1$ impurity coupled through an exchange interaction $J$ with two-degenerate conduction bands. In Fig. 10, we show the NRG conductance of the Kondo model as a function of temperature, for several positive $D$, below and above the critical anisotropy $D_{c}=1.352 \times 10^{-4}(J=0.2, W=1)$. It can be seen that for $D<D_{c}, G(T)$ reaches the unitary limit at low temperatures, corresponding to a fully-screened Kondo effect; on the other hand, for $D>D_{c}$, the conductance goes to zero, as the magnetic moment of the impurity is quenched by the single-ion anisotropy. Close to $D_{c}, G(T)$ exhibits an extended plateau at one-half of the unitary limit value, a typical characteristic of the $2 \mathrm{CK}$ effect.

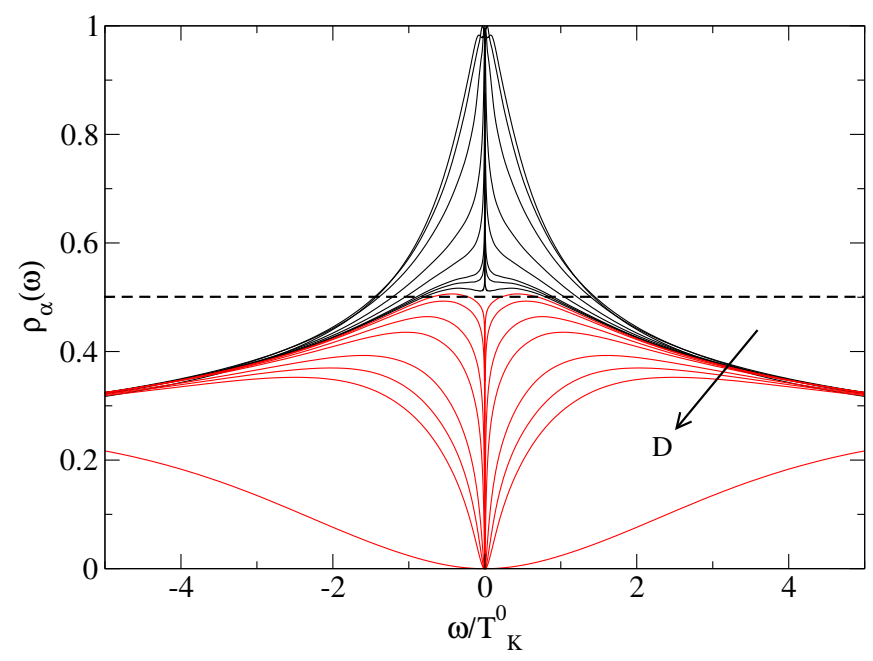

FIG. 11: (Color online) Normalized NRG spectral function $\rho_{\alpha}(\omega)$ of the $S=1$ Kondo impurity model as a function of $T / T_{K}^{0}$, for several positive $D$ across the topological quantum phase transition. The black (red) curves correspond to $D<$ $D_{c}\left(D>D_{c}\right)$. From top to bottom: $D=0.0,5 \times 10^{-5}$, $8 \times 10^{-5}, 1 \times 10^{-4}, 1.15 \times 10^{-4}, 1.2 \times 10^{-4}, 1.21 \times 10^{-4}$, $1.22 \times 10^{-4}, 1.23 \times 10^{-4}, 1.25 \times 10^{-4}, 1.3 \times 10^{-4}, 1.35 \times 10^{-4}$, $1.6 \times 10^{-4}, 1.8 \times 10^{-4}, 2 \times 10^{-4}, 5 \times 10^{-4}$. The parameters are $W=1, J=0.2$. We take $\Lambda=2$ and keep 3000 NRG states. For these parameters, $D_{c}=1.225 \times 10^{-4}$ and $T_{K}^{0}=3.8 \times 10^{-4}$.

As another signature of the quantum phase transition that occurs for $D=D_{c}$, Fig. 11 displays the spectral density of states in the Kondo limit ${ }^{80}$ calculated with NRG of the $S=1$ Kondo impurity model, around the Fermi level energy, for several positive $D$ across the critical one. For $D<D_{c}$ (black curves) the full screening of the $S=1$ impurity gives rise to a Kondo resonance, while for $D>D_{c}$ (red curves) a dip appears at the Fermi level due to the anisotropy-driven quenching of the magnetic degree of freedom of the impurity. At $D \simeq D_{c}$, the spectral density at $\omega=0$ takes half of its value in the fully screened Kondo phase. This is another indication of the emergence of $2 \mathrm{CK}$ physics at critical $D_{c}$.

With the aim of complementing the above mentioned results, now we analyze the behavior of our model (2) with negative $D$. In this case, the isolated impurity has a doubly degenerate ground state, corresponding to $S_{z}=$ \pm 1 . Although these spin projections differ in $\left|\Delta S_{z}\right|=2$ and, consequently, they cannot be connected by the usual second order hybridization processes that originate the Kondo exchange interaction, we have found that fourthorder hybridization processes (see Fig. 12) lead to a fully Kondo screening of the $S=1$ impurity for negative $D$.

The left panel of Fig. (13) displays the NRG impurity 

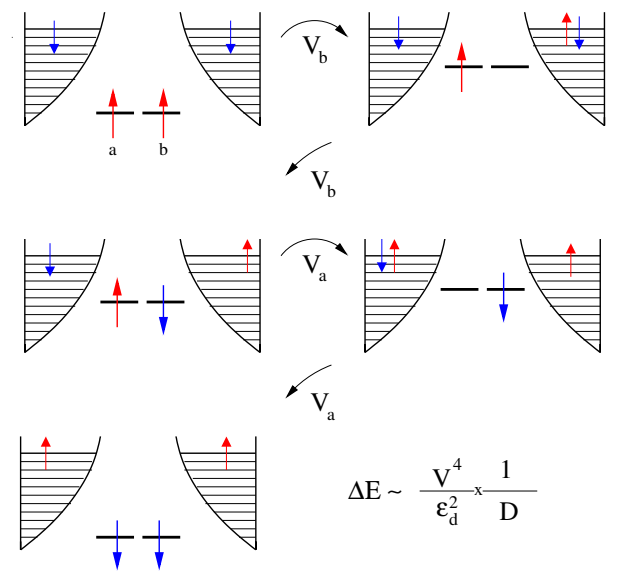

$\Delta \mathrm{E} \sim \frac{\mathrm{V}^{4}}{\varepsilon_{\mathrm{d}}^{2}} \times \frac{1}{\mathrm{D}}$

FIG. 12: (Color online) A fourth-order hybridization process that leads to an effective spin-flip between the $S_{z}= \pm 1$ projections for negative $D$.

entropy contribution as function of the normalized temperature $T / T_{K}^{0}$, for $D=-16 T_{K}^{0}$. It can be seen that, as for $D=0$, at the higher temperatures $e^{S_{i m p}}$ saturates at the value given by the localized Hilbert space. At intermediate temperatures, once the charge fluctuations are frozen, a plateau can be observed with $e^{S_{i m p}} \simeq 2$, corresponding to the double degenerate impurity $S_{z}= \pm 1$ degrees of freedom. As these magnetic states are fully Kondo screened at lower temperatures, the impurity entropy goes to zero. On the other hand, the right panel of Fig. (13) shows the NRG impurity spectral function as function of frequency around the Fermi level, at a very low temperature. $\rho_{\alpha \sigma}$ has the typical structure of a Kondo state, and it can be seen that it satisfies the Friedel sum rule. Both curves, entropy and spectral function, along the NRG spectra, indicates that for negative $D$ the ground state of model (2) is a conventional local Fermi liquid.

Finally, through the NRG conductance (see Fig. (14), we can estimate the Kondo temperature using the usual rule $G\left(T_{K}\right)=G(T \rightarrow 0) / 2$. It can be seen that this energy scale $T_{K}(D)$ rapidly goes down as $D$ becomes more negative. In fact, $T_{K}(D)$ obeys an exponential scaling law with $|D|$ :

$$
T_{K}(D) \propto T_{K}^{0} \exp \left[-c\left(\frac{D}{T_{K}^{0}}\right)^{2}\right]
$$

with $c$ a constant of order of one.

\section{Conclusions}

In this work, we have studied a spin-1 Anderson impurity model -in which the triplet ground state is mixed with a configuration with two doublets by means of two degenerate conduction channels--, that describes a single $\mathrm{Ni}$ impurity embedded in an O-doped $\mathrm{Au}$
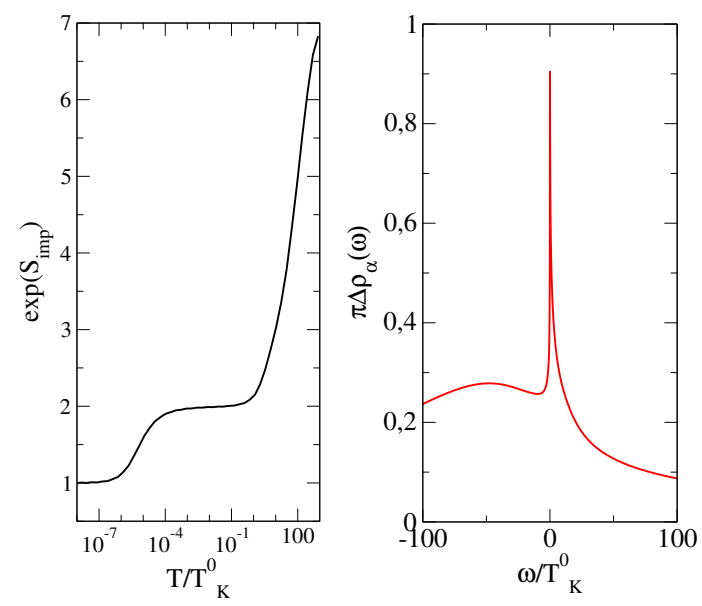

FIG. 13: (Left) NRG impurity entropy $S_{i m p}$ as a function of $T / T_{K}^{0}$. (Right) NRG impurity spectral function $\rho_{\alpha \sigma}(\omega)$ as a function of $\omega / T_{K}^{0}$. The model parameters are $\Delta=0.1$, $\epsilon_{d}=-0.2 \Delta$, with a corresponding $T_{K}^{0} \simeq 1.245 \times 10^{-3}$, and $D=-16 T_{K}^{0}$.

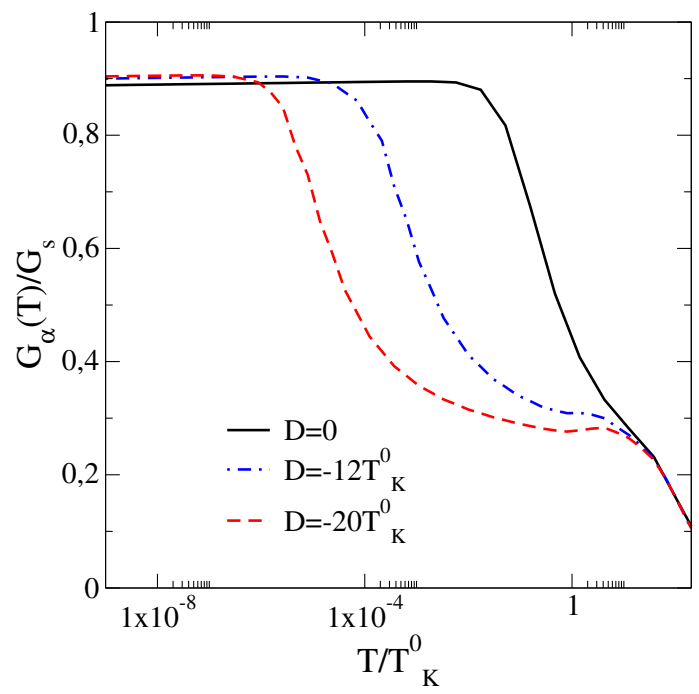

FIG. 14: (Color online) NRG conductance $G_{\alpha}(T)$ as a function of $T / T_{K}^{0}$ for three different negative magnetic anisotropies $D$. The other parameters are the same as in Fig. (13).

chain $^{42}$. In agreement with the predictions of Nozières and Blandin ${ }^{21}$, this two-orbital two-channel impurity model exhibits a fully-screened Kondo effect at low temperatures. As the experimental realizations of the fullyscreened Kondo effect for high-spin quantum dots are rare at present as compared to what happens in bulk systems, our results provide a useful guide to potential experimental studies of the $\mathrm{Ni}$ impurity in gold chains or related systems, allowing, for example, to discern the spin of the impurity. In this sense, to be more realistic, we have included the effect of a single ion anisotropy $D$ in the impurity. There is a plethora of experiments on nanoscopic systems, which cannot be de- 
scribed with the single channel $S=1 / 2$ Anderson (or Kondo) model, in particular several experimental realizations with $S>1 / 2^{14,15,19,24,28,30,33,34}$ and also with degenerate orbitals ${ }^{39-41}$ have been studied. Therefore, it is reasonable to expect that experimental realizations of fully compensated Kondo impurities will appear in the near future.

We have solved the impurity model using two methods that give very reliable information at complementary energy scales: the numerical renormalization group, numerically exact at low energies of the order of the Kondo temperature, and the non-crossing approximation, that takes correct account of the charge transfer processes at higher energies.

For $D=0$, we have found the expected signatures of the local Fermi liquid behavior at low temperatures: the universality with a single Kondo energy scale, the vanishing of the impurity entropy contribution, the large electrical conductance in agreement with the Friedel sum rule, among others. However, the Kondo temperature is strongly reduced in comparison with the $s=1 / 2$ case, a phenomenon that has been experimentally observed in bulk systems since the 1960 's $^{38}$. Furthermore, the empirical expression of the conductance as a function of temperature (Eq. 16), that is used to experimentally discern the spin value of the impurity ${ }^{18}$, has a noticeable different fitting $s$ parameter as compared with the fully screened spin-1/2 and the underscreened spin-1 Kondo cases. Another interesting feature is that the mixed valence regime seems to be much suppressed in the spin-1 case.

At higher energies, the charge transfer peak for the spin-1 model exhibits a very different behavior in comparison with the spin-1/2 model: the Haldane shift of the bare energy $\epsilon_{d}$ is cut in half and has an opposite sign ( $\epsilon_{d}^{*}$ is closer to the Fermi level), while the width of the charge transfer peak is reduced to $\simeq 3 \Delta$. This last result, together with suppression of the mixed valence regime, points out that charge fluctuations are significantly reduced for high-spin impurities.

As found earlier ${ }^{43}$, the single-ion anisotropy $D$ has a strong effect on the Kondo physics: while for any negative $D$ the Kondo effect survives, with a reduced $T_{K}(D)$, for a critical positive $D_{c}$ there is a topological quantum phase transition, from the usual local Fermi liquid at lower $D$ to a topologically non-trivial non-Landau Fermi liquid for larger anisotropies. Just at the transition, the impurity shows the signatures of a non-Fermi liquid two-channel Kondo behavior. In this work we have calculated the conductance and the spectral density in the Kondo limit very near the transition, showing the abrupt remarkable changes of both quantities at $D_{c}$.

For negative $D$ the Kondo effect always persists at low enough temperatures, but the Kondo temperature is strongly reduced because the remaining degenerate states of the impurity are mixed by a higher (fourth) order process in the hybridization between impurity and conduction electrons.

We hope that our detailed study of the spin-1 Ander- son impurity model encourages the experimental search of low dimensional high-spin fully-screened Kondo systems, like the proposed Ni impurity in O-doped Au-chain. As we have shown, there are several observables that can be used to differentiate the high-spin and the usual spin $-1 / 2$ cases.

\section{Acknowledgments}

This work was partially supported by PIP 112-20150100506 of CONICET (Argentina), and PICT 2013-1045 of the ANPCyT (Argentina).

\section{A. Kondo temperature within the NCA approximation}

In this Appendix, we show that the Kondo scale is overestimated within the non-crossing approximation.

In the isotropic case, $D=0$, and for degenerate levels (as for the Ni impurity in the O-doped Au chain), the system of equations that determines the NCA self-energies is reduced to

$$
\begin{aligned}
& \Sigma_{1}(\omega)=\frac{3 \Delta}{2 \pi} \int_{-W}^{W} d \epsilon f(\epsilon) G_{2}(\epsilon+\omega), \\
& \Sigma_{2}(\omega)=\frac{2 \Delta}{\pi} \int_{-W}^{W} d \epsilon f(\epsilon) G_{1}(\omega+\epsilon),
\end{aligned}
$$

where $G_{2}(\omega)$ and $G_{1}(\omega)$ represent the Green's function of the triplet and both doublets $\alpha=x z, y z$ components, respectively.

With the help of the redefinition $\Delta=2 \Delta^{\prime}$ for the hybridization, it can be seen that the set of self-energies equations is the same as for the NCA treatment of the $S U(N) \times S U(M)$ generalization of the multichannel single-impurity Kondo model, with $M=4$ identical conduction bands and being $N=3$ the degeneracy in the impurity spin quantum number ${ }^{81}$,

$$
\begin{aligned}
& \Sigma_{1}(\omega)=\frac{3 \Delta^{\prime}}{\pi} \int_{-W}^{W} d \epsilon f(\epsilon) G_{2}(\epsilon+\omega), \\
& \Sigma_{2}(\omega)=\frac{4 \Delta^{\prime}}{\pi} \int_{-W}^{W} d \epsilon f(\epsilon) G_{1}(\omega+\epsilon) .
\end{aligned}
$$

For this system, the characteristic energy scale $T_{K}^{\mathrm{NCA}}$ can be obtained analytically from the zero temperature limit of the self-energies, and it is given by the expres$\operatorname{sion}^{81,82}$

$$
T_{K}^{\mathrm{NCA}}=W\left(\frac{\Delta^{\prime}}{\pi W}\right)^{\frac{M}{N}} \times \exp \left(\frac{\pi \epsilon_{d}}{N \Delta^{\prime}}\right) .
$$

For the simplest case of the one-channel $(M=1)$, infinite Coulomb repulsion, and $\operatorname{spin} s=1 / 2(N=2)$ Anderson model, NCA gives the Kondo temperature

$$
T_{K, \mathrm{NCA}}^{s=1 / 2}=\sqrt{\frac{\Delta W}{\pi}} e^{\pi \epsilon_{d} / 2 \Delta} .
$$


This value coincides with the exact Kondo energy except for the prefactor $1 / \sqrt{\pi} \approx 0.5$.

On the other hand, for the case of interest we derive the following NCA Kondo scale,

$$
T_{K, N C A}^{S=1}=W\left(\frac{\Delta}{2 \pi W}\right)^{4 / 3} e^{2 \pi \epsilon_{d} / 3 \Delta},
$$

which can be written in terms of $T_{K}^{s=1 / 2}$ and in units of $W$ as follows

$$
T_{K, N C A}^{S=1}=\sqrt{8}\left(\frac{\Delta}{2 \pi}\right)^{2 / 3}\left(T_{K}^{s=1 / 2}\right)^{4 / 3} .
$$

The relation with the asymptotic Kondo temperature for the spin $S=1$ obtained from the NRG calculations in
Eq. (14), $c \sqrt{\Delta}\left(T_{K}^{s=1 / 2}\right)^{2}$, shows that the NCA largely overestimates the Kondo scale as

$$
\frac{T_{K}^{S=1}}{T_{K, N C A}^{S=1}} \approx c(2 \pi)^{4 / 3} \Delta^{1 / 6} e^{\pi \epsilon_{d} / 3 \Delta} \ll 1,
$$

for typical $\epsilon_{d}$ and $\Delta$ values in the Kondo regime.

$$
\begin{gathered}
\text { Intensity of the Kondo peak within the NCA } \\
\text { approximation }
\end{gathered}
$$

For such a model, the NCA spectral density at the Fermi level is expected to be $\rho(0) \sim \frac{2 \pi}{(N+M)^{2} \Delta^{\prime}}$, (see Appendix B of Ref. 82). We have verified that our calculations satisfy this rule (An additional factor 2 was included due to the definition of the physical operator).
1 A. C. Hewson, The Kondo Problem to Heavy Fermions (Cambridge University Press, Cambridge, UK, 1997).

2 J. Kondo, Resistance Minimum in Dilute Magnetic Alloys, Prog. Theor. Phys. 32, 37 (1964).

3 D. Goldhaber-Gordon, H. Shtrikman, D. Mahalu, D. Abusch-Magder, U. Meirav, and M. A. Kastner, Kondo effect in a single-electron transistor, Nature 391, 156 (1998).

4 S. M. Cronenwett, T. H. Oosterkamp, and L. P. Kouwenhoven, A Tunable Kondo Effect in Quantum Dots, Science 281, 540 (1998).

5 D. Goldhaber-Gordon, J. Göres, M. A. Kastner, H. Shtrikman, D. Mahalu, and U. Meirav, From the Kondo Regime to the Mixed-Valence Regime in a Single-Electron Transistor, Phys. Rev. Lett. 81, 5225 (1998).

6 W. G. van der Wiel, S. de Franceschi, T. Fujisawa, J. M. Elzerman, S. Tarucha, and L. P. Kowenhoven, The Kondo Effect in the Unitary Limit, Science 289, 2105 (2000).

7 M. Grobis, I. G. Rau, R. M. Potok, H. Shtrikman, and D. Goldhaber-Gordon, Universal scaling in nonequilibrium transport through a single channel Kondo dot, Phys. Rev. Lett. 100, 246601 (2008).

8 A. V. Kretinin, H. Shtrikman, D. Goldhaber-Gordon, M. Hanl, A. Weichselbaum, J.von Delft, T. Costi, and D. Mahalu, Spin- $\frac{1}{2}$ Kondo effect in an InAs nanowire quantum dot: Unitary limit, conductance scaling, and Zeeman splitting, Phys. Rev. B 84, 245316 (2011).

9 S. Amasha, A. J. Keller, I. G. Rau, A. Carmi, J. A. Katine, H. Shtrikman, Y. Oreg, and D. Goldhaber-Gordon, Pseudospin-Resolved Transport Spectroscopy of the Kondo Effect in a Double Quantum Dot, Phys. Rev. Lett. 110, 046604 (2013).

10 W. Liang, M. P. Shores, M. Bockrath, J. R. Long, and $\mathrm{H}$. Park, Kondo resonance in a single-molecule transistor, Nature 417, 725 (2002).

11 L. H. Yu, Z. K. Keane, J. W. Ciszek, L. Cheng, J. M. Tour, T. Baruah, M. R. Pederson, and D. Natelson, Kondo Resonances and Anomalous Gate Dependence in the Electrical Conductivity of Single-Molecule Transistors, Phys. Rev. Lett. 95, 256803 (2005).

12 M. N. Leuenberger and E. R. Mucciolo, Berry-Phase Oscillations of the Kondo Effect in Single-Molecule Magnets, Phys. Rev. Lett. 97, 126601 (2006).
13 E. A. Osorio, K. O’Neill, M. Wegewijs, N. Stuhr-Hansen, J. Paaske, T. Thomas Bjørnholm, and H. S. J. van der Zant, Electronic Excitations of a Single Molecule Contacted in a Three-Terminal Configuration, Nano. Lett. 7, 3336 (2007).

14 J. J. Parks, A. R. Champagne, G. R. Hutchison, S. FloresTorres, H. D. Abruña, and D. C. Ralph, Tuning the Kondo Effect with a Mechanically Controllable Break Junction, Phys. Rev. Lett. 99, 026601 (2007).

15 N. Roch, S. Florens, V. Bouchiat, W. Wernsdorfer, and F. Balestro, Quantum phase transition in a single-molecule quantum dot, Nature 453, 633 (2008).

16 E. A. Osorio, K. Moth-Poulsen, H. S. J. van der Zant, J. Paaske, P. Hedegård, K. Flensberg, J. Bendix, and T. Thomas Bjørnholm, Electrical Manipulation of Spin States in a Single Electrostatically Gated Transition-Metal Complex, Nano. Lett. 10, 105 (2010).

17 G. D. Scott, Z. K. Keane, J. W. Ciszek, J. M. Tour, and D. Natelson, Universal scaling of nonequilibrium transport in the Kondo regime of single molecule devices, Phys. Rev. B 79, 165413 (2009).

18 J. J. Parks, A. R. Champagne, T. A. Costi, W. W. Shum, A. N. Pasupathy, E. Neuscamman, S. Flores-Torres, P. S. Cornaglia, A. A. Aligia, C. A. Balseiro, G. K.-L. Chan, H. D. Abruña, and D. C. Ralph, Mechanical Control of Spin States in Spin-1 Molecules and the Underscreened Kondo Effect, Science 328, 1370 (2010).

19 S. Florens, A, Freyn, N. Roch, W. Wernsdorfer, F. Balestro, P. Roura-Bas, and A. A. Aligia, Universal transport signatures in two-electron molecular quantum dots: gate-tunable Hund's rule, underscreened Kondo effect and quantum phase transitions, J. Phys. Condens. Matter 23, 243202 (2011), and references therein.

${ }^{20}$ R. Vincent, S. Klyatskaya, M. Ruben, W. Wernsdorfer, and F. Balestro, Electronic read-out of a single nuclear spin using a molecular spin transistor, Nature (London) 488, 357 (2012).

$21 \mathrm{Ph}$. Nozières and A. Blandin, Kondo effect in real metals, J. Physique 41193 (1980).

22 P. Mehta, N. Andrei, P. Coleman, L. Borda, and G. Zaránd, Regular and singular Fermi-liquid fixed points in quantum impurity models, Phys. Rev. B 72, 014430 (2005).

23 E. Sela, A. K. Mitchell, and L. Fritz, Exact Crossover 
Green Function in the Two-Channel and Two-Impurity Kondo Models, Phys. Rev. Lett. 106, 147202 (2011).

24 P. S. Cornaglia, P. Roura-Bas, A. A. Aligia and C. A. Balseiro, Quantum transport through a stretched spin-1 molecule, Europhys. Lett. 93, 47005 (2011).

25 S. Di Napoli, A. Weichselbaum, P. Roura-Bas, A. A. Aligia, Y. Mokrousov, and S. Blügel, Non-Fermi liquid behaviour in transport through Co-doped Au chains, Phys. Rev. Lett. 110, 196402 (2013).

26 S. Di Napoli, P. Roura-Bas, A. Weichselbaum, and A. A. Aligia, Non-Fermi liquid behaviour in nonequilibrium transport through Co-doped Au chains connected to fourfold symmetric leads, Phys. Rev. B 90, 125149 (2014).

27 R. Zitko, R. Peters, and T. Pruschke, Properties of anisotropic magnetic impurities on surfaces, Phys. Rev. B 78, 224404 (2008).

${ }^{28}$ P. Roura Bas and A. A. Aligia, Nonequilibrium transport through a singlet-triplet Anderson impurity, Phys. Rev. B 80, 035308 (2009).

29 D. E. Logan, C. J. Wright, and M. R. Galpin, Correlated electron physics in two-level quantum dots: Phase transitions, transport, and experiment, Phys. Rev. B 80, 125117 (2009).

30 P. Roura-Bas and A. A. Aligia, Nonequilibrium dynamics of a singlet-triplet Anderson impurity near the quantum phase transition, J. Phys.: Condens. Matter 22, 025602 (2010).

31 M. A. Barral, S. Di Napoli, G. Blesio, P. Roura-Bas, A. Camjayi, L. O. Manuel, and A. A. Aligia, Kondo behavior and conductance through 3 d impurities in gold chains doped with oxygen, J. Chem. Phys. 146, 092315 (2017).

32 D. Jacob, M. Soriano, J. J. Palacios, Kondo effect and spin quenching in high-spin molecules on metal substrates Phys. Rev. B 88, 134417 (2013).

33 R. Tuerhong, F.O. Ngassam, S. Watanabe, J. Onoe, M. Alouani, amd J.P. Bucher, Two-Dimensional Organometallic Kondo Lattice with Long-Range Antiferromagnetic Order, J. Phys. Chem. C 122, 20046 (2018).

34 M. Ormaza, P. Abufager, B. Verlhac, N. Bachellier, M.-L. Bocquet, N. Lorente, and L. Limot, Controlled spin switching in a metallocene molecular junction Nature Commun. 8, 1974 (2017).

35 Y. Wang, X. Li, X. Zheng, and J. Yang, Electronic and magnetic properties of $\mathrm{CoPc}$ and $\mathrm{FePc}$ molecules on graphene: the substrate, defect, and hydrogen adsorption effects, Phys. Chem. Chem. Phys. 21, 5424 (2019).

36 Y. Wang, X. Li, X. Zheng, and J. Yang, Manipulation of spin and magnetic anisotropy in bilayer magnetic molecular junctions, Phys. Chem. Chem. Phys. 20, 26396 (2018).

37 S. Di Napoli , M. A. Barral, P. Roura-Bas, A. M. Llois, and A. A. Aligia, Unusual Kondo physics in a Co impurity atom embedded in noblemetal chains, IEEE Trans. Magn. 494683 (2013).

38 A. H. Nevidomskyy and P. Coleman, Kondo resonance narrowing in $d$ - and $f$-electron systems, Phys. Rev. Lett. 103, 147205 (2009).

39 E. Minamitani, N. Tsukahara, D. Matsunaka, Y. Kim, N. Takagi, and M. Kawai, Symmetry-Driven Novel Kondo Effect in a Molecule Phys. Rev. Lett. 109, 086602 (2012).

40 J. Fernández, A. A. Aligia, and A. M. Lobos, Valence fluctuations in a lattice of magnetic molecules: Application to iron(II) phtalocyanine molecules on Au(111) Europhys. Lett. 109, 37011 (2015).
41 M. Moro-Lagares, J. Fernández, P. Roura-Bas, M. R. Ibarra, A. A. Aligia, and D. Serrate Quantifying the leading role of the surface state in the Kondo effect of Co/Ag(111) Phys. Rev. B 97, 235442 (2018).

42 S. Di Napoli, M. A. Barral, P. Roura-Bas, L. O. Manuel, A. M. Llois, and A. A. Aligia, Kondo physics in a Ni impurity embedded in O-doped Au chains, Phys. Rev. B 92, 085120 (2015).

43 G. G. Blesio, L. O. Manuel, P. Roura-Bas, and A. A. Aligia, Topological quantum phase transition between Fermi liquid phases in an Anderson impurity model, Phys. Rev. B 98, 195425 (2018).

44 M. D. Daybell and W. A. Steyert, Localized magnetic impurity states in metals: some experimental relationships, Rev. Mod. Phys. 40, 380 (1968).

45 C. Payen, E. Janod, K. Schoumacker, C. D. Batista, K. Hallberg and A. A. Aligia, Evidence of quantum criticality in the doped Haldane system $\mathrm{Y}_{2} \mathrm{BaNiO}_{5}$, Phys. Rev. B 62, 2998 (2000); references therein.

46 R. Zitko, NRG-LJUBLJANA, open source numerical renormalization group code, http://nrgljubljana.ijs.si.

47 R. Zitko and T. Pruschke, Energy resolution and discretization artifacts in the numerical renormalization group, Phys. Rev. B 79, 085106 (2009).

48 A. A. Aligia, Effect of covalency and interactions on the trigonal splitting in $\mathrm{Na}_{x} \mathrm{CoO}_{2}$, Phys. Rev. B 88, 075128 (2013), and references therein.

49 A. M. Oleś, Antiferromagnetism and correlation of electrons in transition metals, Phys. Rev. B 28, 327 (1983).

${ }^{50}$ O. Sakai, Y. Shimizu, and T. Kasuya, Single-particle and magnetic excitation spectra of degenerate Anderson model with finite $f-f$ Coulomb interaction, J. Phys. Soc Jpn. $\mathbf{5 8}, 3666$ (1989);

51 W. Izumida, O. Sakai, and Y. Shimizu, Kondo effect in single quantum dot systems - Study with numerical renormalization group method, J. Phys. Soc Jpn. 67, 2444 (1998).

52 M. Koyima, S. Yotsuhashi, and K. Miyake, Numerical renormalization group study of two-level Kondo effect: Discovery of new fixed point, Acta Phys. Pol. B 22, 1331 (2003).

53 L. De Leo and M. Fabrizio, Spectral properties of a twoorbital Anderson impurity model across a non-Fermi-liquid fixed point, Phys. Rev. B 69, 245114 (2004).

54 A. K. Zhuravlev, V. Y. Irkhin, M. I. Katsnelson, and A. I. Lichtenstein, Kondo Resonance for Orbitally Degenerate Systems, Phys. Rev. Lett. 93, 236403 (2004).

55 Y. Nishikawa and A. C. Hewson, Hund's rule coupling in models of magnetic impurities and quantum dots, Phys. Rev. B 86, 245131 (2012).

56 T. Pruschke and R. Bulla, Hund's coupling and the metalinsulator transition in the two-band Hubbard model, Eur. Phys. J. B 44, 217 (2005).

57 N.E. Bickers, Review of techniques in the large- $N$ expansion for dilute magnetic alloys, Rev. of Mod. Phys. 59, 845 (1987).

58 A. A. Aligia, P. Roura-Bas, and S. Florens, Impact of capacitance and tunneling asymmetries on Coulomb blockade edges and Kondo peaks in nonequilibrium transport through molecular quantum dots, Phys. Rev. B 92, 035404 (2015).

59 J. Fernández, F. Lisandrini, P. Roura-Bas, C. Gazza, and A. A. Aligia, Width of the charge-transfer peak in the $S U(N)$ impurity Anderson model and its relevance to nonequilibrium transport, Phys. Rev. B 97, 045144 (2018).

60 Th. Pruschke and N. Grewe, The Anderson model with 
finite Coulomb repulsion, Z. Phys. B 74, 439 (1989).

61 D. E. Logan, M. P. Eastwood, and M. A. Tusch, A local moment approach to the Anderson model, J. Phys. Condens. Matter 10, 2673 (1998).

62 J. Könemann, B. Kubala, J. König, and R. J. Haug, Tunneling resonances in quantum dots: Coulomb interaction modifies the width, Phys. Rev. B 73, 033313 (2006).

${ }^{63}$ N. S. Wingreen and Y. Meir, Anderson model out of equilibrium: Noncrossing-approximation approach to transport through a quantum dot, Phys. Rev. B 49, 11040 (1994).

${ }^{64}$ L. Tosi, P. Roura-Bas, and A. A. Aligia. Restoring the $S U(4)$ Kondo regime in a double quantum dot system, J. Phys.: Condens. Matter 27335601 (2015).

65 F. D. M. Haldane, Scaling theory of the asymmetric Anderson model, Phys. Rev. Lett. 40, 416 (1978).

${ }^{66}$ L. Vaugier, A.A. Aligia, and A.M. Lobos, Spectral density of an interacting dot coupled indirectly to conducting leads, Phys. Rev. B 76, 165112 (2007).

${ }^{67} \mathrm{P}$. Roura-Bas, Universal scaling in transport out of equilibrium through a single quantum dot using the non-crossing approximation, Phys. Rev. B 81, 155327 (2010).

68 D. P. Daroca, P. Roura-Bas, and A. A. Aligia, Relation between width of zero-bias anomaly and Kondo temperature in transport measurements through correlated quantum dots: Effect of asymmetric coupling to the leads, Phys. Rev. B 98, 245406 (2018)

69 T. A. Costi, Kondo effect in a magnetic field and the magnetoresistivity of Kondo alloys, Phys. Rev. Lett. 85, 1504 (2000).

70 T. A. Costi, L. Bergqvist, A. Weichselbaum, J. von Delft, T. Micklitz, A. Rosch, P. Mavropoulos, P. H. Dederichs, F. Mallet, L. Saminadayar, and C. Baüerle, Kondo Decoherence: Finding the Right Spin Model for Iron Impurities in Gold and Silver, Phys. Rev. Lett. 102, 056802 (2009)

71 T. A. Costi and V. Zlatić, Thermoelectric transport through strongly correlated quantum dots, Phys. Rev. B 81, 235127 (2010)

72 N. Roch, S. Florens, T. A. Costi, W. Wernsdorfer, and F.
Balestro, Observation of the Underscreened Kondo Effect in a Molecular Transistor Phys. Rev. Lett. 103, 197202 (2009).

73 A. F. Otte, M. Ternes, K. von Bergmann, S. Loth, H. Brune, C. P. Lutz, C. F. Hirjibehedin, and A. J. Heinrich, The role of magnetic anisotropy in the Kondo effect, Nat. Phys. 4, 847 (2008).

74 J. C. Oberg, M. R. Calvo, F. Delgado, M. Moro-Lagares, D. Serrate, D. Jacob, J. Fernandez-Rossier, and C. F. Hirjibehedin, Control of single-spin magnetic anisotropy by exchange coupling, Nat. Nanotechnol. 9, 64 (2013).

75 B. W. Heinrich, L. Braun, J. I. Pascual, and K. J. Franke, Tuning the magnetic anisotropy of single molecules, Nano. Lett. 15, 4024 (2015).

76 R. Hiraoka, E. Minamitani, R. Arafune, N. Tsukahara, S. Watanabe, M. Kawai, and N. Takagi, Single-molecule quantum dot as a Kondo simulator, Nature Commun. 8, 16012 (2017).

77 D. Jacob, Renormalization of single-ion magnetic anisotropy in the absence of the Kondo effect, Phys. Rev. B 97, 075428 (2018).

78 O. J. Curtin, Y. Nishikawa, A. C. Hewson, and D. J. G. Crow, Fermi liquids and the Luttinger theorem, J. Phys. Commun. 2, 031001 (2018).

${ }^{79} \mathrm{~K}$. Seki and S. Yunoki, Topological interpretation of the Luttinger theorem, Phys. Rev. B 96, 085124 (2017).

80 A. K. Mitchell and D. E. Logan, Two-channel Kondo phases and frustration-induced transitions in triple quantum dots, Phys. Rev. B 81, 075126 (2010).

81 D. L. Cox and A. E. Ruckenstein, Spin-flavor separation and non-Fermi-liquid behavior in the multichannel Kondo problem: A large-N approach, Phys. Rev. Lett. 71, 1613 (1993).

82 T. S. Kim and D. L. Cox, One-, two-, and three-channel Kondo effects for a model Ce $e^{3+}$ impurity in a metal, Phys. Rev. B 5512594 (1997). 